\title{
Ground State Asymptotics of a Dilute, Rotating Gas
}

\author{
Robert Seiringer \\ Department of Physics, Jadwin Hall, Princeton University \\ P.O. Box 708, Princeton, NJ 08544, USA \\ Email: rseiring@math.princeton.edu
}

June 3, 2003

\begin{abstract}
We investigate the ground state properties of a gas of interacting particles confined in an external potential in three dimensions and subject to rotation around an axis of symmetry. We consider the socalled Gross-Pitaevskii (GP) limit of a dilute gas. Analyzing both the absolute and the bosonic ground state of the system we show, in particular, their different behavior for a certain range of parameters. This parameter range is determined by the question whether the rotational symmetry in the minimizer of the GP functional is broken or not. For the absolute ground state, we prove that in the GP limit a modified GP functional depending on density matrices correctly describes the energy and reduced density matrices, independent of symmetry breaking. For the bosonic ground state this holds true if and only if the symmetry is unbroken.
\end{abstract}

\section{Introduction}

Since the first experimental realization of Bose-Einstein condensation (BEC) in dilute gases of alkali atoms [1, 2], much interest has been devoted to the study of their rotational properties. Beautiful results showing the appearance of vortices and the formation of vortex arrays have been obtained in various experiments [3, 4. Many of the striking features of rotating Bose-Einstein 
condensates are well described by means of the Gross-Pitaevskii (GP) functional, and most theoretical investigations rely on the approximation made in its use (see, e.g., [5, 6]).

Recently a rigorous justification of the GP functional for non-rotating systems has been obtained [7], and a proof of BEC in the ground state was given in [8]. These results do not extend to the case of a rotating system in a simple way, however. In this paper, we investigate the effect of the rotation. Several new features come into play. First, Bose statistics become essential. While for the case of a non-rotating system the ground state of the Hamiltonian is automatically symmetric in the particle coordinates, this in not necessarily the case for rotating systems and, in fact, is shown not to be the case for the system under consideration here (at least for a certain parameter range). That is, it is important to distinguish the absolute ground state from the bosonic ground state (the ground state of the Hamiltonian restricted to totally symmetric wave functions), the two having significantly different physical properties. Secondly, the appearance of vortices breaks the rotational symmetry, leading to non-uniqueness of the minimizer of the GP functional (see Theorem 1 below). This makes it necessary to study a generalized GP functional, which depends on density matrices rather than densities alone. These two properties are in fact related, as we show, the symmetry being broken if and only if the absolute and bosonic ground state energy differ by a significant amount (of the order of the energy itself).

The main result of this paper is that the generalized GP density matrix functional mentioned above correctly describes the absolute ground state of the Hamiltonian under consideration here, in a certain dilute limit. More precisely, it gives the correct asymptotics of the ground state energy and all the $n$-particle reduced density matrices of the ground state of the system. In the case of unbroken rotational symmetry, the GP density matrix functional agrees with the usual GP functional, and these results apply also to the bosonic ground state. This extends previous results on superfluidity in 9]. In the general case of broken symmetry, however, we are not able to establish the precise asymptotics in the bosonic case, but we give bounds on the corresponding energy that show in particular that it's energy differs from the absolute ground state energy by a significant amount. Moreover, this implies that the absolute ground state has a huge degeneracy that increases exponentially with the particle number.

Before we can give the precise formulation of the preceding statements, we have to define the system under consideration. For $\Omega \in \mathbb{R}^{3}$ and a realvalued 
$V \in L_{\text {loc }}^{\infty}\left(\mathbb{R}^{3}, d \boldsymbol{x}\right)$ let $H_{0}$ be the one-particle Hamiltonian

$$
H_{0}=-\Delta-\boldsymbol{\Omega} \cdot \boldsymbol{L}+V(\boldsymbol{x}),
$$

acting on $L^{2}\left(\mathbb{R}^{3}, d \boldsymbol{x}\right)$. Here $\Delta=\boldsymbol{\nabla}^{2}$ is the Laplacian on $\mathbb{R}^{3}$, and $\boldsymbol{L}=-i \boldsymbol{x} \wedge \boldsymbol{\nabla}$ denotes the angular momentum operator. The operator $H_{0}$ is the appropriate Hamiltonian for one particle confined in a trap potential $V$ and rotating with angular velocity $\Omega$, in the rotating frame. To ensure that $H_{0}$ is semibounded from below, we assume that

$$
\left[V(\boldsymbol{x})-\frac{\omega^{2}}{4} r^{2}\right]_{-} \in L^{\infty}\left(\mathbb{R}^{3}\right)
$$

for all $\omega$ in some non-zero interval $\left[0, \Omega_{c}\right) \subset \mathbb{R}_{+}$, where we denote by $[\cdot]_{-}$ the negative part, and $r$ denotes the radial distance perpendicular to $\Omega$, i.e., $r=|\boldsymbol{x} \wedge \Omega| /|\Omega|$. We call the largest possible $\Omega_{c}$ the critical angular velocity, allowing it to be infinity. Writing $H_{0}$ as

$$
H_{0}=(-i \boldsymbol{\nabla}-\boldsymbol{A})^{2}+V(\boldsymbol{x})-\frac{|\boldsymbol{\Omega}|^{2}}{4} r^{2}
$$

where $\boldsymbol{A}=\frac{1}{2} \boldsymbol{\Omega} \wedge \boldsymbol{x}$ is the vector potential of a constant magnetic field in the direction of $\Omega$, we see that $H_{0}$ is semibounded from below for $|\Omega|<\Omega_{c}$, and unbounded for $|\Omega|>\Omega_{c}$. We will henceforth always assume that $|\Omega|<\Omega_{c}$. Note that the condition (1.2) on $V$, as well as $\Omega_{c}$, depend on the direction of $\Omega$, which we assume to be fixed throughout the paper, whereas its absolute value is allowed to vary.

We also assume that $\lim _{|\boldsymbol{x}| \rightarrow \infty} V(\boldsymbol{x})=\infty$ uniformly in all directions, implying discrete spectrum of $H_{0}$. Moreover, we demand that $V$ commutes with $\boldsymbol{\Omega} \cdot \boldsymbol{L}$, or, in other words, it is axially symmetric, being a function that depends only on $r$ and $z=\boldsymbol{x} \cdot \boldsymbol{\Omega} /|\boldsymbol{\Omega}|$. Without loss of generality $V \geq 0$.

To define the $N$-particle problem, consider the Hilbert space $\mathcal{H}_{N}=$ $\bigotimes_{i=1}^{N} L^{2}\left(\mathbb{R}^{3}, d \boldsymbol{x}_{i}\right)$, the $N$-fold tensor product of the one-particle space. The Hamiltonian corresponding to $N$ particles in a trap $V$, rotating with angular velocity $\Omega$, and interacting pairwise with an interaction potential $v$, is given by

$$
H_{N, \boldsymbol{\Omega}, a}=\sum_{i=1}^{N} H_{0}^{(i)}+\sum_{1 \leq i<j \leq N} \frac{1}{a^{2}} v\left(\left(\boldsymbol{x}_{i}-\boldsymbol{x}_{j}\right) / a\right)
$$


Here the superscript $(i)$ means that $H_{0}$ acts on the $i$ 'th part in the tensor product. The interaction potential $v$ is assumed to positive, spherically symmetric and of compact support. We do not demand it to be integrable, it is allowed to have a hard core, which reduces the domain of definition of $H_{N, \boldsymbol{\Omega}, a}$ to wave functions in $\mathcal{H}_{N}$ that vanish whenever two particles are closer together then the size of the hard core. The positive parameter $a$ appearing in $H_{N, \Omega, a}$ determines the range of the interaction $v$. We assume that $v$ has scattering length 1 (see [7] or [10] for a definition), implying that $a^{-2} v(\boldsymbol{x} / a)$ has scattering length $a$.

We are interested in the ground state properties of $H_{N, \boldsymbol{\Omega}, a}$ for large $N$ and small $a$. In particular, the Gross-Pitaevskii limit $a \sim N^{-1}$ will be investigated. Its significance comes from the fact that it ensures that the contributions to the energy of all the terms in the Hamiltonian (1.4) are of the same order as $N \rightarrow \infty$. Let $E^{\mathrm{QM}}$ denote the ground state energy of $H_{N, \Omega, a}$, i.e.,

$$
E^{\mathrm{QM}}(N, \boldsymbol{\Omega}, a)=\inf \operatorname{spec} H_{N, \boldsymbol{\Omega}, a} .
$$

Besides $N, \boldsymbol{\Omega}$ and $a$ it depends on $V$ and $v$, of course, but these potentials are assumed to be fixed once and for all. From the discussion above it is clear that $E^{\mathrm{QM}}$ is finite for $|\Omega|<\Omega_{c}$, and that $E^{\mathrm{QM}}(N, \Omega, a)=E^{\mathrm{QM}}(N,-\boldsymbol{\Omega}, a)$.

A different problem is obtained by restricting ourselves to wave functions in $\mathcal{H}_{N}$ that are totally symmetric with respect to exchange of two particle coordinates, which corresponds to assuming the particles to be Bosons. We denote the corresponding ground state energy by $E_{\text {bose }}^{\mathrm{QM}}(N, \boldsymbol{\Omega}, a)$, i.e.,

$$
E_{\text {bose }}^{\mathrm{QM}}(N, \Omega, a)=\inf \left\{\left\langle\Psi \mid H_{N, \boldsymbol{\Omega}, a} \Psi\right\rangle: \Psi \in P_{\text {bose }} \mathcal{H}_{N},\|\Psi\|_{2}=1\right\},
$$

where $P_{\text {bose }}$ denotes the projection onto totally symmetric functions. The two quantities (1.5) and (1.6) a a priori unrelated, except for the trivial inequality $E^{\mathrm{QM}}(N, \boldsymbol{\Omega}, a) \leq E_{\text {bose }}^{\mathrm{QM}}(N, \boldsymbol{\Omega}, a)$. For $\boldsymbol{\Omega}=\mathbf{0}$, i.e., the case of a Schrödinger operator, it is well known that $E^{\mathrm{QM}}(N, \mathbf{0}, a)=E_{\text {bose }}^{\mathrm{QM}}(N, \mathbf{0}, a)$, but for $\boldsymbol{\Omega} \neq \mathbf{0}$ this need not necessarily be the case. In fact it will turn out that, at least for certain values of the parameters, the two quantities are different.

Before we can state our main results, we have to introduce some functionals which will turn out to be related to the asymptotic behavior of the $N$-particle problem defined by $H_{N, \boldsymbol{\Omega}, a}$ for large $N$ and small $a$. We do this in the next section. 


\section{Gross-Pitaevskii Functionals}

We define the Gross-Pitaevskii density matrix (DM) functional to be

$$
\mathcal{E}_{\boldsymbol{\Omega}, g}^{\mathrm{DM}}[\gamma]=\operatorname{Tr}\left[H_{0} \gamma\right]+4 \pi g \int \rho_{\gamma}(\boldsymbol{x})^{2} d \boldsymbol{x}
$$

Here $\gamma$ is a one-particle density matrix, a positive trace-class operator on $L^{2}\left(\mathbb{R}^{3}, d \boldsymbol{x}\right)$, and $\rho_{\gamma}$ denotes its density. The corresponding ground state energy, the infimum of (2.1) under the condition $\operatorname{Tr}[\gamma]=1$, will be denoted by $E^{\mathrm{DM}}(\boldsymbol{\Omega}, g)$, i.e.,

$$
E^{\mathrm{DM}}(\boldsymbol{\Omega}, g)=\inf \left\{\mathcal{E}_{\boldsymbol{\Omega}, g}^{\mathrm{DM}}[\gamma]: \operatorname{Tr}[\gamma]=1\right\} .
$$

Note that one could equivalently define the ground state energy under the subsidiary condition $\operatorname{Tr}[\gamma]=N$, or any other constant, but this can be easily related to (2.2) by a trivial scaling of $E^{\mathrm{DM}}$ and $g$. Again it is clear that $E^{\mathrm{DM}}(\Omega, g)$ is finite for $|\Omega|<\Omega_{c}$ and $g \geq 0$, which we will always assume.

The analogue of the DM functional for a two-dimensional gas was introduced in [11] as a generalization of the standard GP functional, which is given by restricting $\mathcal{E}_{\boldsymbol{\Omega}, g}^{\mathrm{DM}}$ to density matrices of rank one. Equivalently, one can write

$$
\mathcal{E}_{\boldsymbol{\Omega}, g}^{\mathrm{GP}}[\phi]=\left\langle\phi \mid H_{0} \phi\right\rangle+4 \pi g \int|\phi(\boldsymbol{x})|^{4} d \boldsymbol{x}
$$

for functions $\phi \in L^{2}\left(\mathbb{R}^{3}, d \boldsymbol{x}\right)$, and define the corresponding ground state energy as

$$
E^{\mathrm{GP}}(\boldsymbol{\Omega}, g)=\inf \left\{\mathcal{E}_{\boldsymbol{\Omega}, g}^{\mathrm{GP}}[\phi]:\|\phi\|_{2}=1\right\} .
$$

Since one-dimensional projections are legitimate density matrices in (2.1), it is clear that $E^{\mathrm{DM}}(\boldsymbol{\Omega}, g) \leq E^{\mathrm{GP}}(\boldsymbol{\Omega}, g)$.

In the case of two dimensions both the functionals $\mathcal{E}_{\boldsymbol{\Omega}, g}^{\mathrm{DM}}$ and $\mathcal{E}_{\boldsymbol{\Omega}, g}^{\mathrm{GP}}$ were studied in [11]. Many of the results translate directly to the three-dimensional case, with minor modifications, and we merely state them here in the following two propositions, omitting the proofs.

PROPOSITION 1 (Minimizer of $\mathcal{E}_{\boldsymbol{\Omega}, \boldsymbol{g}}^{\mathrm{DM}}$ ). For each $0 \leq|\Omega|<\Omega_{c}$ and $g>0$ there exists a unique minimizing density matrix for (2.1) under the condition $\operatorname{Tr}[\gamma]=1$, denoted by $\gamma_{\boldsymbol{\Omega}, g}^{\mathrm{DM}}$. This minimizer also minimizes the linearized functional

$$
\gamma \mapsto \operatorname{Tr}\left[\left(H_{0}+8 \pi g \rho_{\boldsymbol{\Omega}, g}^{\mathrm{DM}}\right) \gamma\right]
$$


(under the same normalization condition), where $\rho_{\boldsymbol{\Omega}, g}^{\mathrm{DM}}$ denotes the density of $\gamma_{\boldsymbol{\Omega}, g}^{\mathrm{DM}}$. Moreover, $\gamma_{\boldsymbol{\Omega}, g}^{\mathrm{DM}}$ has finite rank. Its density $\rho_{\boldsymbol{\Omega}, g}^{\mathrm{DM}}$ is a bounded function, with $\rho_{\boldsymbol{\Omega}, g}^{\mathrm{DM}}(\boldsymbol{x}) \leq \mu_{\boldsymbol{\Omega}, g}^{\mathrm{DM}} /(8 \pi g)$, where $\mu_{\boldsymbol{\Omega}, g}^{\mathrm{DM}}$ is the chemical potential in DM theory, which is the ground state energy of (2.5).

Note that for uniqueness $g>0$ is essential, since the ground state of $H_{0}$ may be degenerate, implying non-uniqueness for $g=0$.

Because of uniqueness, we also know that $\rho_{\boldsymbol{\Omega}, g}^{\mathrm{DM}}$ is axially symmetric, with symmetry axis $\boldsymbol{\Omega}$. Note that the uniqueness of $\gamma_{\boldsymbol{\Omega}, g}^{\mathrm{DM}}$ is non-trivial. From the strict convexity of $\mathcal{E}_{\boldsymbol{\Omega}, g}^{\mathrm{DM}}$ in $\rho_{\gamma}$ it follows only that $\rho_{\boldsymbol{\Omega}, g}^{\mathrm{DM}}$ is unique, i.e., that every possible minimizer has the same density. By examining the ground state space of the operator appearing in (2.5) one then shows that there can be only one density matrix with the property that it minimizes (2.5) and has $\rho_{\boldsymbol{\Omega}, g}^{\mathrm{DM}}$ as its density.

For $\mathcal{E}_{\boldsymbol{\Omega}, g}^{\mathrm{GP}}$ essentially the same results as in Prop. 1 are true, except for uniqueness.

PROPOSITION 2 (Minimizers of $\mathcal{E}_{\Omega, g}^{\mathrm{GP}}$ ). For each $0 \leq|\Omega|<\Omega_{c}$ and $g \geq 0$ there exists a minimizing function for (2.3) under the condition $\|\phi\|_{2}=$ 1. Any minimizer $\phi$ fulfills the GP equation

$$
H_{0} \phi+8 \pi g|\phi|^{2} \phi=\mu_{\boldsymbol{\Omega}, g}^{\mathrm{GP}} \phi
$$

where $\mu_{\boldsymbol{\Omega}, g}^{\mathrm{GP}}=\mu_{\boldsymbol{\Omega}, g}^{\mathrm{GP}}(\phi)$ is the chemical potential, given by

$$
\mu_{\boldsymbol{\Omega}, g}^{\mathrm{GP}}=E^{\mathrm{GP}}(\boldsymbol{\Omega}, g)+4 \pi g \int|\phi(\boldsymbol{x})|^{4} d \boldsymbol{x} .
$$

One might suspect that the two minimization problems (2.2) and (2.4) are equivalent, in the sense that the minimizer of $\mathcal{E}_{\boldsymbol{\Omega}, g}^{\mathrm{DM}}$ has rank one, i.e, $\gamma_{\boldsymbol{\Omega}, g}^{\mathrm{DM}}$ is a one-dimensional projection onto the minimizer of $\mathcal{E}_{\boldsymbol{\Omega}, g}^{\mathrm{GP}}$, which would consequently have to be unique (up to a constant phase factor, of course). However, for $\boldsymbol{\Omega} \neq \mathbf{0}$ this is not true, at least not for $g$ large enough. The following Theorem 1 is the analogue of Theorem 4 and Corollary 2 in [1], where the case of a two-dimensional system was considered. Since its proof is not a simple generalization of the two-dimensional case, it will be given in the Appendix. Due to some technical complications it is more difficult to prove this theorem in three dimensions, and it will be convenient not to consider the most general external potentials $V$, but to restrict ourselves to a special class with sufficiently nice properties. The general class of $V$ 's which our 
proof applies to is rather difficult to characterize, but sufficient conditions are easy to state. We assume them for simplicity, they are general enough to allow for a quite large class of $V$ 's, but they are by no means necessary for Theorem 1 to hold, as the proof in the appendix shows.

For Theorem 1 (and only there) we will assume that the external potential $V$ fulfills the bounds

$$
V(\boldsymbol{x}) \leq \text { const. }\left(1+r^{s}+|z|^{p}\right)
$$

and

$$
V(\boldsymbol{x}) \geq \text { const. }\left(r^{s}+|z|^{p}\right)-\text { const. }
$$

for suitable constants (independent of $r$ and $z$ ) and for some exponents $2 \leq$ $s<\infty$ and $0<p<\infty$. Note that $s \geq 2$ is necessary for condition (1.2) to hold.

THEOREM 1 (Non-equivalence of $\mathcal{E}_{\boldsymbol{\Omega}, \boldsymbol{g}}^{\mathrm{GP}}$ and $\mathcal{E}_{\boldsymbol{\Omega}, \boldsymbol{g}}^{\mathrm{DM}}$ ). Assume that $V$ satisfies the bounds (2.8) and (2.9). For any $0<|\Omega|<\Omega_{c}$ there exists a $g_{\mid \boldsymbol{\Omega}} \mid$ such that $g \geq g_{|\boldsymbol{\Omega}|}$ implies that no minimizer of $\mathcal{E}_{\boldsymbol{\Omega}, g}^{\mathrm{GP}}$ is an eigenfunction of the angular momentum $\boldsymbol{\Omega} \cdot \boldsymbol{L}$, and consequently the minimizer is not unique. Moreover, for $g \geq g_{|\boldsymbol{\Omega}|}, E^{\mathrm{GP}}(\boldsymbol{\Omega}, g)>E^{\mathrm{DM}}(\boldsymbol{\Omega}, g)$, and the minimizer $\gamma_{\boldsymbol{\Omega}, g}^{\mathrm{DM}}$ of $\mathcal{E}_{\boldsymbol{\Omega}, g}^{\mathrm{DM}}$ has at least rank 2.

For a given direction of $\Omega$, we will denote by $\Xi \subset\left[0, \Omega_{c}\right) \times \mathbb{R}_{+}$the set of parameters where $\mathcal{E}_{\boldsymbol{\Omega}, g}^{\mathrm{GP}}$ and $\mathcal{E}_{\boldsymbol{\Omega}, g}^{\mathrm{DM}}$ are not equivalent, i.e.,

$$
\Xi \equiv\left\{(|\boldsymbol{\Omega}|, g): E^{\mathrm{GP}}(\boldsymbol{\Omega}, g)>E^{\mathrm{DM}}(\boldsymbol{\Omega}, g)\right\}
$$

This is the case if and only if the rank of $\gamma_{\boldsymbol{\Omega}, g}^{\mathrm{DM}}$ is greater or equal to two, and therefore, by Prop. 11 the ground state of $H_{0}+8 \pi g \rho_{\boldsymbol{\Omega}, g}^{\mathrm{DM}}$ is degenerate. Note that Theorem 1 states that $\Xi$ is non-empty, at least for external potentials satisfying the bounds (2.8) and (2.9).

In the non-rotating case, i.e., $\boldsymbol{\Omega}=\mathbf{0}, E^{\mathrm{GP}}(\mathbf{0}, g)$ and $E^{\mathrm{DM}}(\mathbf{0}, g)$ are equal for all $g \geq 0$. This remains true if $|\Omega|$ is not too large. In fact one can show that there exists an $\Omega_{g}>0$, depending on $g$, such that $E^{\mathrm{GP}}(\boldsymbol{\Omega}, g)=$ $E^{\mathrm{DM}}(\Omega, g)$ for $|\Omega| \leq \Omega_{g}[11]$.

Note that both $E^{\mathrm{GP}}$ and $E^{\mathrm{DM}}$ are concave functions of their parameters and, in particular, continuous. Hence $\Xi$ is an open set, being the complement of the zero set of the continuous function $E^{\mathrm{GP}}(\Omega, g)-E^{\mathrm{DM}}(\Omega, g)$, and both axis $g=0$ and $|\Omega|=0$ are not contained in $\Xi$. 


\section{Main Results}

With the preliminaries of the previous section in hand, we can now state our main results. We are interested in the ground state energy $E^{\mathrm{QM}}(N, \boldsymbol{\Omega}, a)$ of the $N$-particle Hamiltonian (1.4), for large $N$ and small $a$. In fact it turns out that $a \sim N^{-1}$ is the case of interest. As explained in the Introduction, $a \sim$ $N^{-1}$ implies that all terms in the Hamiltonian (1.4) yield a contribution to the ground state energy of the same order as $N \rightarrow \infty$. That is, for some fixed $g>0$, we will set $a=g N^{-1}$, or, more generally, we will assume that $N a \rightarrow g$ as $N \rightarrow \infty$. Moreover, we will also derive results for the corresponding ground states or, more generally, for approximate ground states. We call a sequence $\Psi_{N} \in \mathcal{H}_{N}$ an approximate ground state if $\left\|\Psi_{N}\right\|_{2}=1$ and

$$
\lim _{N \rightarrow \infty, N a \rightarrow g}\left\langle\Psi_{N} \mid H_{N, \boldsymbol{\Omega}, a} \Psi_{N}\right\rangle E^{\mathrm{QM}}(N, \boldsymbol{\Omega}, a)^{-1}=1
$$

for fixed $\Omega$ and $g$. Given such an approximate ground state, we define its $n$-particle reduced density matrix by the kernel

$$
\begin{aligned}
& \Gamma_{N}^{(n)}\left(\boldsymbol{x}_{1}, \ldots,\right.\left.\boldsymbol{x}_{n}, \boldsymbol{y}_{1}, \ldots, \boldsymbol{y}_{n}\right)=\frac{1}{N !} \sum_{\pi \in S_{N}} \int_{\mathbb{R}^{3(N-n)}} \prod_{j=n+1}^{N} d \boldsymbol{x}_{j} \\
& \times \Psi_{N}\left(\pi\left(\boldsymbol{x}_{1}, \ldots, \boldsymbol{x}_{N}\right)\right) \Psi_{N}^{*}\left(\pi\left(\boldsymbol{y}_{1}, \ldots, \boldsymbol{y}_{n}, \boldsymbol{x}_{n+1}, \ldots, \boldsymbol{x}_{N}\right)\right)
\end{aligned}
$$

where $S_{N}$ denotes the permutation group and $\pi\left(\boldsymbol{x}_{1}, \ldots, \boldsymbol{x}_{N}\right)$ is a permutation of the $N$ variables $\boldsymbol{x}_{i}, 1 \leq i \leq N$. The ${ }^{*}$ denotes complex conjugation. The $\Gamma_{N}^{(n)}$ defined in (3.2) are trace class operators on $\mathcal{H}_{n}$, and the normalization is chosen such that $\operatorname{Tr}\left[\Gamma_{N}^{(n)}\right]=1$. Our main result on the properties of the absolute ground state of $H_{N, \boldsymbol{\Omega}, a}$ is the following.

THEOREM 2 (Ground state asymptotics). For given $\boldsymbol{\Omega}$ and $g$ let $\gamma_{\boldsymbol{\Omega}, g}^{\mathrm{DM}}$ be the unique minimizer of $\mathcal{E}_{\boldsymbol{\Omega}, g}^{\mathrm{DM}}$, with corresponding energy $E^{\mathrm{DM}}(\boldsymbol{\Omega}, g)$. Let $\Gamma_{N}^{(n)}$ denote the n-particle reduced density matrix of an approximate ground state of $H_{N, \Omega, a}$. Then

$$
\lim _{N \rightarrow \infty} \frac{1}{N} E^{\mathrm{QM}}\left(N, \boldsymbol{\Omega}, g N^{-1}\right)=E^{\mathrm{DM}}(\boldsymbol{\Omega}, g)
$$

uniformly in $g$ on compact intervals in $(0, \infty)$, and, for each $n \in \mathbb{N}$,

$$
\lim _{N \rightarrow \infty} \Gamma_{N}^{(n)}=\underbrace{\gamma_{\boldsymbol{\Omega}, g}^{\mathrm{DM}} \otimes \cdots \otimes \gamma_{\boldsymbol{\Omega}, g}^{\mathrm{DM}}}_{n \text { times }}
$$


in the usual norm of trace class operators on $\bigotimes_{i=1}^{n} L^{2}\left(\mathbb{R}^{3}, d \boldsymbol{x}_{i}\right)$.

This theorem will be proved in the next section. Concerning the bosonic ground state energy $E_{\text {bose }}^{\mathrm{QM}}(N, \boldsymbol{\Omega}, g)$, we cannot give the precise asymptotics as in (3.3), but we can give upper and lower bounds. One might conjecture that (3.5) below holds as equality, but we cannot prove this. However, the conjecture is supported by the following theorem.

THEOREM 3 (Asymptotics for bosonic ground state energy). For fixed $\Omega$ and $g$ we have that

$$
\limsup _{N \rightarrow \infty} \frac{1}{N} E_{\text {bose }}^{\mathrm{QM}}\left(N, \Omega, g N^{-1}\right) \leq E^{\mathrm{GP}}(\Omega, g)
$$

Moreover,

$$
\liminf _{N \rightarrow \infty} \frac{1}{N} E_{\text {bose }}^{\mathrm{QM}}\left(N, \Omega, g N^{-1}\right)>E^{\mathrm{DM}}(\Omega, g)
$$

if and only if

$$
E^{\mathrm{GP}}(\boldsymbol{\Omega}, g)>E^{\mathrm{DM}}(\boldsymbol{\Omega}, g),
$$

i.e., $(|\Omega|, g) \in \Xi$, and the same is true with liminf replaced by limsup in Eq. (3.6).

Remark 1. Inequality (3.6) holds uniformly in $g$ for compact intervals of $g$ in $\Xi$. More precisely, for any $\varepsilon>0$ such that the closed interval $[(|\Omega|, g-$ $\varepsilon),(|\Omega|, g+\varepsilon)]$ is contained in $\Xi$,

$$
\liminf _{N \rightarrow \infty} \inf _{g^{\prime} \in(g-\varepsilon, g+\varepsilon)}\left\{\frac{1}{N} E_{\text {bose }}^{\mathrm{QM}}\left(N, \boldsymbol{\Omega}, g^{\prime} N^{-1}\right)-E^{\mathrm{DM}}\left(\boldsymbol{\Omega}, g^{\prime}\right)\right\}>0 .
$$

This property will be important in the proof of Corollary 1 .

Remark 2. Theorems 2 and 3 together have the following consequences on the Bose gas. If $(|\Omega|, g) \notin \Xi$, e.g., $E^{\mathrm{GP}}(\Omega, g)=E^{\mathrm{DM}}(\boldsymbol{\Omega}, g)$, there is a unique minimizer of the GP functional (2.3). Moreover,

$$
\lim _{N \rightarrow \infty} \frac{1}{N} E_{\text {bose }}^{\mathrm{QM}}\left(N, \boldsymbol{\Omega}, g N^{-1}\right)=E^{\mathrm{GP}}(\boldsymbol{\Omega}, g)
$$

as can be seen from the lower bound (3.3) and the upper bound (3.5). Therefore the bosonic ground state is an approximate ground state for the unrestricted problem, and hence (3.4) holds. The density matrix $\gamma_{\boldsymbol{\Omega}, g}^{\mathrm{DM}}$ in this case 
is the one-dimensional projection onto the minimizer of $\mathcal{E}_{\boldsymbol{\Omega}, g}^{\mathrm{GP}}$, and (3.4) proves complete Bose-Einstein condensation of all $n$-particle density matrices. For $\boldsymbol{\Omega}=\mathbf{0}$ this was proved in $[\mathrm{8}$. The persistence of BEC for $\boldsymbol{\Omega} \neq \mathbf{0}$ can also be interpreted as a superfluid behavior of the system (see [9]).

Note that these results are true not only for the bosonic ground state, but for any approximate bosonic ground state. Moreover, the notion of approximate ground states is readily generalized to $N$-particle density matrices. In particular, the assertions above are true for all Gibbs states

$$
\Gamma_{N, \boldsymbol{\Omega}, a}^{\beta} \equiv \frac{P_{\text {bose }} \exp \left(-\beta H_{N, \boldsymbol{\Omega}, a}\right)}{\operatorname{Tr}\left[P_{\text {bose }} \exp \left(-\beta H_{N, \boldsymbol{\Omega}, a}\right)\right]},
$$

with $\beta>0$, assuming that the trace is finite, which is guaranteed for external potentials $V$ that increase at least logarithmically in $|\boldsymbol{x}|$ at infinity. We will show in Sect. 4.3 that

$$
\lim _{N \rightarrow \infty} \frac{1}{N}\left(\operatorname{Tr}\left[H_{N, \boldsymbol{\Omega}, g N^{-1}} \Gamma_{N, \boldsymbol{\Omega}, g N^{-1}}^{\beta}\right]-E_{\mathrm{bose}}^{\mathrm{QM}}\left(N, \boldsymbol{\Omega}, g N^{-1}\right)\right)=0
$$

for all $\beta>0$, implying, for $(|\Omega|, g) \notin \Xi$, complete BEC of the reduced density matrices of (3.10), i.e., they converge to the right hand side of (3.4), where $\gamma_{\boldsymbol{\Omega}, g}^{\mathrm{DM}}$ is now the projection onto the unique GP minimizer. Note, however, that fixing $\beta$ is really a zero-temperature limit, since the relevant temperature scale depends on the (mean) density, which goes to infinity in our limit. To obtain a true effect of the temperature one has to scale it appropriately with $N$. E.g., for a harmonic trap potential the relevant temperature scale would be $T \sim N^{1 / 3}[6]$.

Note that for (3.11) to hold true it is essential to restrict oneselves to the bosonic subspace in (3.10). Without this restriction (3.11) (with $E_{\text {bose }}^{\mathrm{QM}}$ replaced by $E^{\mathrm{QM}}$ ) will not be true, as can be seen from the non-interacting case $g=0$.

A simple corollary of Theorem 3 is the non-uniqueness of ground states of the Hamiltonian $H_{N, \boldsymbol{\Omega}, a}$. Even more is true, namely the ground state degeneracy grows exponentially with $N$.

COROLLARY 1 (Ground state degeneracy). For $(|\boldsymbol{\Omega}|, g) \in \Xi$ and $N$ large enough, no ground state of $H_{N, \Omega, g N^{-1}}$ has bosonic symmetry. Moreover, if $\mathcal{N}(N,|\Omega|, a)$ denotes the multiplicity of the ground state of $H_{N, \boldsymbol{\Omega}, a}$, and $(|\Omega|, g) \in \Xi$, then

$$
\liminf _{N \rightarrow \infty} \frac{1}{N} \ln \mathcal{N}\left(N,|\Omega|, g N^{-1}\right)>0
$$


Remark 3. Corollary 1 states that the degeneracy of the ground state of $H_{N, \boldsymbol{\Omega}, g N^{-1}}$ grows at least exponentially with $N$ if $(|\Omega|, g) \in \Xi$. If the external potential $V$ does not grow too slowly at infinity, it is not difficult to see that in fact $\mathcal{N}\left(N,|\Omega|, g N^{-1}\right)$ grows also at most exponentially. Namely, if we assume that $\operatorname{Tr}\left[\exp \left(-\beta H_{0}\right)\right]<\infty$ for some $\beta>0$, which is guaranteed if $V$ has at least logarithmic increase in $|\boldsymbol{x}|$, then

$$
\limsup _{N \rightarrow \infty} \frac{1}{N} \ln \mathcal{N}\left(N,|\Omega|, g N^{-1}\right) \leq \ln \operatorname{Tr}\left[\exp \left(\beta\left(E^{\mathrm{DM}}(\Omega, g)-H_{0}\right)\right)\right] \text {. }
$$

This is shown in Sect. 4.4.

The proofs of the results stated in this section can be found in Section 4 below. The energy difference of the bosonic system to the one without symmetry restrictions can be understood as being due to the positive correlation energy that gets added when trying to symmetrize a state where not essentially all the particles occupy the same state, as is the case in the regime of symmetry breaking, where the minimizer of $\mathcal{E}^{\mathrm{DM}}$ has at least rank 2 . It may therefore be favorable for all bosons to occupy the same state, restoring the complete BEC but breaking the rotational symmetry by choosing one of the minimizers of the GP functional.

All the results in this paper refer to three-dimensional systems, but analogous results can be obtained also for two-dimensional systems, as has been shown, in the non-rotating case, in [12]. The DM and GP functionals in this case have been studied in [11. Moreover, we could also allow for internal degrees of freedom of the particles, e.g. spin, which would not affect the results on the absolute ground state, but the ones on the bosonic ground state. In this case the GP functional has to be replaced by the DM functional restricted to density matrices of a definite rank equal to the number of internal states (compare with Section 6 in [1] ).

\section{Proofs}

Before we give the proof of the results stated in the previous section, we state in Section 4.1 two auxiliary Lemmas that will be used later. The proof of Theorem 2 is given in Section 4.2, and the proof of Theorem 3, together with the assertions made in Remarks 1 and 2, in Section 4.3. Finally, Corollary 1 and the subsequent Remark 3 are proved in Section 4.4. 


\subsection{Preliminaries}

The following Lemma is needed in the proof of Theorem 2 below. It is related to the generalized Poincaré inequalities studied in [13]. For a measurable set $\mathcal{O} \subset \mathbb{R}^{3}$ we denote by $\mathcal{O}^{c}$ its complement, and by $|\mathcal{O}|$ its Lebesgue measure.

LEMMA 1. Let $\boldsymbol{A} \in L_{\mathrm{loc}}^{2}\left(\mathbb{R}^{3} ; \mathbb{R}^{3}\right)$ and $V \in L_{\mathrm{loc}}^{\infty}\left(\mathbb{R}^{3} ; \mathbb{R}\right)$, and assume that $\lim _{|\boldsymbol{x}| \rightarrow \infty} V(\boldsymbol{x})=\infty$. Let

$$
E=\inf \operatorname{spec}\left[(-i \boldsymbol{\nabla}-\boldsymbol{A}(\boldsymbol{x}))^{2}+V(\boldsymbol{x})\right],
$$

and let $P$ denote the projector in $\mathcal{H}=L^{2}\left(\mathbb{R}^{3}, d \boldsymbol{x}\right)$ onto the corresponding ground states. Let

$$
\Delta E=\inf \operatorname{spec}\left[(-i \boldsymbol{\nabla}-\boldsymbol{A}(\boldsymbol{x}))^{2}+V(\boldsymbol{x})\right] \uparrow_{(1-P) \mathcal{H}}-E
$$

denote the gap in the spectrum above the ground state energy, which is positive because of the discrete spectrum of the operator under consideration.

For all $\varepsilon>0$ there exists a $\delta>0$ such that for all $\mathcal{O} \subset \mathbb{R}^{3}$ with $\left|\mathcal{O}^{c}\right|<\delta$ and for all $f \in \mathcal{H}$

$$
\begin{aligned}
\varepsilon \int_{\mathbb{R}^{3}}|(i \boldsymbol{\nabla}+\boldsymbol{A}) f|^{2}+\int_{\mathcal{O}}|(i \boldsymbol{\nabla}+\boldsymbol{A}) f|^{2}+\int_{\mathbb{R}^{3}} V|f|^{2} & \\
& \geq E\|f\|_{L^{2}\left(\mathbb{R}^{3}\right)}^{2}+\Delta E\|f-P f\|_{L^{2}\left(\mathbb{R}^{3}\right)}^{2} .
\end{aligned}
$$

Proof. It is no restriction to assume that $V \geq 0$. As in [13] we will use a compactness argument. Suppose that the Lemma is wrong. Then there exists an $\varepsilon_{0}>0$ and a sequence of pairs $\left(f_{n}, \mathcal{O}_{n}\right)$, such that $\lim _{n \rightarrow \infty}\left|\mathcal{O}_{n}^{c}\right|=0$, $\left\|f_{n}\right\|_{L^{2}\left(\mathbb{R}^{3}\right)}=1$, and

$$
\begin{aligned}
\lim _{n \rightarrow \infty}\left[\varepsilon_{0} \int_{\mathbb{R}^{3}}\left|(i \boldsymbol{\nabla}+\boldsymbol{A}) f_{n}\right|^{2}\right. & +\int_{\mathcal{O}_{n}}\left|(i \boldsymbol{\nabla}+\boldsymbol{A}) f_{n}\right|^{2} \\
& \left.+\int_{\mathbb{R}^{3}} V\left|f_{n}\right|^{2}-\Delta E\left\|f_{n}-P f_{n}\right\|_{L^{2}\left(\mathbb{R}^{3}\right)}^{2}\right] \leq E
\end{aligned}
$$

Now both $(i \boldsymbol{\nabla}+\boldsymbol{A}) f_{n}$ and $f_{n}$ are bounded sequences in $L^{2}\left(\mathbb{R}^{3}\right)$, so we can pass to a subsequence that converges weakly in $L^{2}\left(\mathbb{R}^{3}\right)$ to $(i \boldsymbol{\nabla}+\boldsymbol{A}) f$ and $f$, respectively. We may also assume that $\sum_{n}\left|\mathcal{O}_{n}^{c}\right|$ is finite. Defining $\Sigma_{N}$ 
by $\Sigma_{N}=\mathbb{R}^{3} \backslash \bigcup_{n \geq N} \mathcal{O}_{n}^{c}$ we have $\Sigma_{N} \subset \mathcal{O}_{n}$ for $n \geq N$. Using weak lower semicontinuity of the norms in question, we therefore get

$$
\begin{array}{r}
\liminf _{n \rightarrow \infty}\left[\varepsilon_{0} \int_{\mathbb{R}^{3}}\left|(i \boldsymbol{\nabla}+\boldsymbol{A}) f_{n}\right|^{2}+\int_{\mathcal{O}_{n}}\left|(i \boldsymbol{\nabla}+\boldsymbol{A}) f_{n}\right|^{2}+\int_{\mathbb{R}^{3}} V\left|f_{n}\right|^{2}\right] \\
\geq \sup _{N}\left[\varepsilon_{0} \int_{\mathbb{R}^{3}}|(i \boldsymbol{\nabla}+\boldsymbol{A}) f|^{2}+\int_{\Sigma_{N}}|(i \boldsymbol{\nabla}+\boldsymbol{A}) f|^{2}+\int_{\mathbb{R}^{3}} V|f|^{2}\right] \\
=\left[\left(1+\varepsilon_{0}\right) \int_{\mathbb{R}^{3}}|(i \boldsymbol{\nabla}+\boldsymbol{A}) f|^{2}+\int_{\mathbb{R}^{3}} V|f|^{2}\right]>E \int_{\mathbb{R}^{3}}|f|^{2} .
\end{array}
$$

Now since $V$ goes to infinity at infinity, $-\Delta+V$ has a compact resolvent (cf., e.g., [14, Thm. XIII.65]), and hence also $(i \boldsymbol{\nabla}+\boldsymbol{A})^{2}+V$ has a compact resolvent [15, Thm. 2.7]. Since

$$
\int_{\mathbb{R}^{3}}\left(\left|(i \boldsymbol{\nabla}+\boldsymbol{A}) f_{n}\right|^{2}+V\left|f_{n}\right|^{2}\right)<C
$$

for some $C<\infty$ independent of $n$, we can conclude that $f_{n}$ is contained in a compact subset of $L^{2}\left(\mathbb{R}^{3}\right)$, and thus $f_{n} \rightarrow f$ strongly in $L^{2}\left(\mathbb{R}^{3}\right)$. This implies that $\|f\|_{2}=1$, and also

$$
\lim _{n \rightarrow \infty}\left\|f_{n}-P f_{n}\right\|_{L^{2}\left(\mathbb{R}^{3}\right)}=\|f-P f\|_{L^{2}\left(\mathbb{R}^{3}\right)} .
$$

Together with (4.5) this contradicts (4.4).

Using more sophisticated methods, as in [13], it is possible to investigate the relation between $\varepsilon$ and $\delta$. This is needed to get precise error estimates, but we shall not do this here.

In the proof of Theorem 2 it will be necessary to study a homogeneous gas of $n$ particles, described by the Hamiltonian

$$
-\sum_{i=1}^{n} \Delta_{i}+\sum_{1 \leq i<j \leq n} \frac{1}{a^{2}} v\left(\left(\boldsymbol{x}_{i}-\boldsymbol{x}_{j}\right) / a\right),
$$

acting on $L^{2}\left(\Lambda^{n}\right)$. The particles are confined to $\Lambda=[0, L]^{3}$, a box of side length $L$, and we use Neumann boundary conditions. A lower bound to the ground state energy of (4.8) was obtained in [16], and we shall describe the 
result here. In fact, the lower bound in [16] was obtained via a lower bound to the expression

$$
\sum_{i=1}^{n}\left[\frac{\varepsilon}{2} \int_{\Lambda^{n}}\left|\boldsymbol{\nabla}_{i} f\right|^{2}+(1-\varepsilon) \int_{K_{i}}\left|\boldsymbol{\nabla}_{i} f\right|^{2}\right]+\sum_{i<j} \int_{\Lambda^{n}} \frac{1}{a^{2}} v\left(\left(\boldsymbol{x}_{i}-\boldsymbol{x}_{j}\right) / a\right)|f|^{2}
$$

where $0<\varepsilon<1$ and $K_{i} \subset \Lambda^{n}$ is given by

$$
K_{i}=\left\{\left(\boldsymbol{x}_{1}, \ldots, \boldsymbol{x}_{n}\right) \in \Lambda^{n}: \min _{k, k \neq i}\left|\boldsymbol{x}_{i}-\boldsymbol{x}_{k}\right| \leq R\right\}
$$

for some $R>0$. This is exactly the expression that we have to bound from below in the proof of Theorem 2, see Eq. (4.26). The result is the following. It is valid for all spherically symmetric $v \geq 0$ with finite range and scattering length 1.

LEMMA 2. Let $E_{\varepsilon}(n, L)$ denote the infimum of $(4.9)$ over all functions $f \in L^{2}\left(\Lambda^{n}\right)$ with $\|f\|_{2}=1$, and let $Y=a^{3} n / L^{3}$. If $R \geq a Y^{-5 / 17}, \varepsilon \geq Y^{1 / 17}$, and $n \geq Y^{-1 / 17}$, then there exists a constant $C>0$ such that

$$
E_{\varepsilon}(n, L) \geq 4 \pi a \frac{n^{2}}{L^{3}}\left(1-C Y^{1 / 17}\right)(1-\varepsilon) \text {. }
$$

The proof can be found in [16] (see also [17] for a more elaborate discussion). Strictly speaking, it was derived for the expression (4.9) with $\varepsilon / 2$ replaced by $\varepsilon$ in front of the first term, but it is easy to see that this additional factor does not affect the main result, only the constant appearing in (4.11). Note that the condition on $n$ means that $n \geq(L / a)^{1 / 6}$.

\subsection{Proof of Theorem 2}

We start by considering the case $n=2$ in (3.4). In order to be able to obtain information about the two-particle density matrix, we start by introducing a modified DM functional. Let $U \in C_{0}^{\infty}\left(\mathbb{R}^{3} \times \mathbb{R}^{3}\right)$ be realvalued and symmetric, i.e., $U(\boldsymbol{x}, \boldsymbol{y})=U(\boldsymbol{y}, \boldsymbol{x})$, and let $\delta \in \mathbb{R}$. The modified DM functional is defined as

$$
\widetilde{\mathcal{E}}^{\mathrm{DM}}[\gamma]=\operatorname{Tr}\left[H_{0} \gamma\right]+4 \pi g \int \rho_{\gamma}(\boldsymbol{x})^{2} d \boldsymbol{x}+\delta \int U(\boldsymbol{x}, \boldsymbol{y}) \rho_{\gamma}(\boldsymbol{x}) \rho_{\gamma}(\boldsymbol{y}) d \boldsymbol{x} d \boldsymbol{y}
$$


We suppress the dependence on the parameters for simplicity of notation. We assume that $\delta$ is small enough such that $\widetilde{\mathcal{E}}^{\mathrm{DM}}$ is still strictly convex in $\rho_{\gamma}$, which is in particular the case for $\delta\|U\|_{L^{2}\left(\mathbb{R}^{6}\right)}<4 \pi g$. The ground state energy of (4.12) will be denoted by $\widetilde{E}^{\mathrm{DM}}$, which depends, for fixed $U$, on $\Omega$, $g$ and $\delta$. As for the DM functional with $\delta=0$, one can use standard methods to show the existence of a minimizing density matrix for $\widetilde{\mathcal{E}}^{\text {DM }}$. Uniqueness is not clear, however, but this is of no concern to us. (For $\delta$ small enough, $\widetilde{\mathcal{E}}^{\mathrm{DM}}$ is strictly convex in $\rho_{\gamma}$, and therefore the density of a minimizer is unique. This convexity property will be important in the proof of Lemma 4 below.) Let $\widetilde{\gamma}$ denote a minimizer of $\widetilde{\mathcal{E}}^{\mathrm{DM}}$, with corresponding density $\widetilde{\rho}$. One can show that $\widetilde{\rho}$ is a bounded, continuously differentiable function that decreases exponentially at infinity. Moreover, as in Prop. 1 , $\widetilde{\gamma}$ also minimizes the linear functional

$$
\gamma \mapsto \operatorname{Tr}[\widetilde{H} \gamma]
$$

with $\widetilde{H}$ given by

$$
\widetilde{H}=H_{0}+8 \pi g \widetilde{\rho}(\boldsymbol{x})+2 \delta \int U(\boldsymbol{x}, \boldsymbol{y}) \widetilde{\rho}(\boldsymbol{y}) d \boldsymbol{y} .
$$

That is, the range of $\widetilde{\gamma}$ is contained in the span of the ground states of $\widetilde{H}$, whose ground state energy is given by

$$
\inf \operatorname{spec} \widetilde{H}=\widetilde{E}^{\mathrm{DM}}(\boldsymbol{\Omega}, g, \delta)+4 \pi g \int \widetilde{\rho}(\boldsymbol{x})^{2} d \boldsymbol{x}+\delta \int U(\boldsymbol{x}, \boldsymbol{y}) \widetilde{\rho}(\boldsymbol{x}) \widetilde{\rho}(\boldsymbol{y}) d \boldsymbol{x} d \boldsymbol{y}
$$

It is important to note that $\widetilde{E}^{\mathrm{DM}}$ is differentiable in $\delta$ at $\delta=0$. This follows from concavity in $\delta$ and the fact that the minimizer at $\delta=0$ is unique (cf., e.g., [18). The derivative is given by

$$
\left.\frac{\partial \widetilde{E}^{\mathrm{DM}}(\boldsymbol{\Omega}, g, \delta)}{\partial \delta}\right|_{\delta=0}=\int U(\boldsymbol{x}, \boldsymbol{y}) \rho_{\boldsymbol{\Omega}, g}^{\mathrm{DM}}(\boldsymbol{x}) \rho_{\boldsymbol{\Omega}, g}^{\mathrm{DM}}(\boldsymbol{y}) d \boldsymbol{x} d \boldsymbol{y}
$$

We will now evaluate upper and lower bounds on the ground state energy of the $N$-particle problem. We also add our auxiliary potential $U$ to the Hamiltonian, and estimate in the following the quantity

$$
E^{\mathrm{QM}}(N, \boldsymbol{\Omega}, a, \delta)=\inf \operatorname{spec}\left[H_{N, \boldsymbol{\Omega}, a}+\frac{2 \delta}{N} \sum_{1 \leq i<j \leq N} U\left(\boldsymbol{x}_{i}, \boldsymbol{x}_{j}\right)\right]
$$


We start with the upper bound. It is derived by analogous considerations as in [7, but instead of using a trial vector we use an $N$-particle trial density matrix, whose kernel has the form

$$
\Gamma\left(\boldsymbol{x}_{1}, \ldots, \boldsymbol{x}_{N}, \boldsymbol{y}_{1}, \ldots, \boldsymbol{y}_{N}\right)=F\left(\boldsymbol{x}_{1}, \ldots, \boldsymbol{x}_{N}\right) F\left(\boldsymbol{y}_{1}, \ldots, \boldsymbol{y}_{N}\right) \prod_{i=1}^{N} \widetilde{\gamma}\left(\boldsymbol{x}_{i}, \boldsymbol{y}_{i}\right)
$$

where $\widetilde{\gamma}$ is a minimizer of (4.12), and $F$ is the Dyson wave function defined in [7. It is given by

$$
F\left(\boldsymbol{x}_{1}, \ldots, \boldsymbol{x}_{N}\right)=\prod_{i=1}^{N} f\left(t_{i}\left(\boldsymbol{x}_{1}, \ldots, \boldsymbol{x}_{i}\right)\right)
$$

where $t_{i}=\min \left\{\left|\boldsymbol{x}_{i}-\boldsymbol{x}_{j}\right|, 1 \leq j \leq i-1\right\}$ is the distance of $\boldsymbol{x}_{i}$ to its nearest neighbor among the points $\boldsymbol{x}_{1}, \ldots, \boldsymbol{x}_{i-1}$, and $f$ is a function of $t \geq 0$. It is chosen to be

$$
f(t)=\left\{\begin{array}{cl}
f_{0}(t) / f_{0}(b) & \text { for } t<b \\
1 & \text { for } t \geq b
\end{array}\right.
$$

where $f_{0}$ is the solution of the zero energy scattering equation for the interaction potential, i.e., $-\Delta f+\frac{1}{2} a^{-2} v(\boldsymbol{x} / a) f=0$, and $b$ is some cut-off parameter of order $b \sim N^{-1 / 3}$. The function $F$ is a suitable generalization of the function Dyson used in [19] to obtain an upper bound on the ground state energy of a homogeneous Bose gas of hard spheres. The calculation of the upper bound follows along the same lines as in [7], with the result that

$$
E^{\mathrm{QM}}\left(N, \boldsymbol{\Omega}, g N^{-1}, \delta\right) \leq N \widetilde{E}^{\mathrm{DM}}(\Omega, g, \delta)\left(1+O\left(N^{-2 / 3}\right)\right)
$$

uniformly in $g$ on compact intervals.

We now proceed with the lower bound. Fix some $R>0$ and $0<\varepsilon<1$. They will be chosen later to depend on $N$ in a definite way. We introduce the short hand notation

$$
\boldsymbol{X}_{i}=\left(\boldsymbol{x}_{1}, \ldots, \boldsymbol{x}_{i-1}, \boldsymbol{x}_{i+1}, \ldots, \boldsymbol{x}_{N}\right)
$$

and

$$
d \boldsymbol{X}_{i}=\prod_{j=1, j \neq i}^{N} d \boldsymbol{x}_{j}
$$


Moreover, let $\boldsymbol{A}(\boldsymbol{x})=\frac{1}{2} \boldsymbol{\Omega} \times \boldsymbol{x}$, and let $r_{i}$ denote the radial distance of $\boldsymbol{x}_{i}$ orthogonal to $\Omega$. Let $\nabla_{j}=\nabla_{\boldsymbol{x}_{j}}$. Given any $\Psi \in \mathcal{H}_{N}$ with $\|\Psi\|_{2}=1$, we can write the expectation value of our modified Hamiltonian as

$$
\left\langle\Psi\left|H_{N, \boldsymbol{\Omega}, a}+\frac{2 \delta}{N} \sum_{i<j} U\left(\boldsymbol{x}_{i}, \boldsymbol{x}_{j}\right)\right| \Psi\right\rangle=\sum_{j=1}^{N}\left(E_{j}^{(1)}+E_{j}^{(2)}\right),
$$

where

$$
\begin{aligned}
E_{j}^{(1)}=\int_{\mathbb{R}^{3(N-1)}} d \boldsymbol{X}_{j}\left[(1-\varepsilon) \int_{\mathcal{O}_{j}^{c}} d \boldsymbol{x}_{j}\left|\left(i \boldsymbol{\nabla}_{j}+\boldsymbol{A}\left(\boldsymbol{x}_{j}\right)\right) \Psi\right|^{2}\right. \\
+\frac{\varepsilon}{2} \int_{\mathbb{R}^{3}} d \boldsymbol{x}_{j}\left|\left(i \boldsymbol{\nabla}_{j}+\boldsymbol{A}\left(\boldsymbol{x}_{j}\right)\right) \Psi\right|^{2} \\
\left.+\int_{\mathbb{R}^{3}} d \boldsymbol{x}_{j}\left(V\left(\boldsymbol{x}_{j}\right)-\frac{|\boldsymbol{\Omega}|^{2}}{4} r_{j}^{2}+8 \pi g \widetilde{\rho}\left(\boldsymbol{x}_{j}\right)+2 \delta \int U\left(\boldsymbol{x}_{j}, \boldsymbol{y}\right) \widetilde{\rho}(\boldsymbol{y}) d \boldsymbol{y}\right)|\Psi|^{2}\right]
\end{aligned}
$$

and

$$
\begin{aligned}
& E_{j}^{(2)}=\int_{\mathbb{R}^{3(N-1)}} d \boldsymbol{X}_{j}[(1-\varepsilon) \int_{\mathcal{O}_{j}} d \boldsymbol{x}_{j}\left|\left(i \boldsymbol{\nabla}_{j}+\boldsymbol{A}\left(\boldsymbol{x}_{j}\right)\right) \Psi\right|^{2} \\
&+\frac{\varepsilon}{2} \int_{\mathbb{R}^{3}} d \boldsymbol{x}_{j}\left|\left(i \boldsymbol{\nabla}_{j}+\boldsymbol{A}\left(\boldsymbol{x}_{j}\right)\right) \Psi\right|^{2} \\
&-\int_{\mathbb{R}^{3}} d \boldsymbol{x}_{j}\left(8 \pi g \widetilde{\rho}\left(\boldsymbol{x}_{j}\right)+2 \delta \int U\left(\boldsymbol{x}_{j}, \boldsymbol{y}\right) \widetilde{\rho}(\boldsymbol{y}) d \boldsymbol{y}\right)|\Psi|^{2} \\
&\left.+\frac{1}{2} \sum_{i=1, i \neq j}^{N} \int_{\mathbb{R}^{3}} d \boldsymbol{x}_{j}\left(\frac{1}{a^{2}} v\left(\left(\boldsymbol{x}_{i}-\boldsymbol{x}_{j}\right) / a\right)+\frac{2 \delta}{N} U\left(\boldsymbol{x}_{i}, \boldsymbol{x}_{j}\right)\right)|\Psi|^{2}\right] .
\end{aligned}
$$

We choose, for fixed $\boldsymbol{X}_{j}$,

$$
\mathcal{O}_{j}=\left\{\boldsymbol{x}_{j} \in \mathbb{R}^{3}: \min _{k, k \neq j}\left|\boldsymbol{x}_{j}-\boldsymbol{x}_{k}\right| \leq R\right\} .
$$

In the following, we will investigate the two terms (4.25) and (4.26) separately. The results are formulated in the following Lemmas. It is always understood that, for fixed $g, N a-g=o(1)$ as $N \rightarrow \infty$, and that $\delta$ is small enough, as explained in the beginning of Section 4.2 . 
LEMMA 3. Let $\widetilde{P}$ denote the projector onto the ground states of $\widetilde{H}$, defined in 4.14$)$. If $R \ll O\left(N^{-1 / 3}\right)$ one can choose $O\left(N^{-2 / 17}\right) \ll \varepsilon \leq o(1)$ as $N \rightarrow \infty$ such that

$$
\begin{aligned}
\sum_{j=1}^{N} E_{j}^{(1)} & \geq N\left(\widetilde{E}^{\mathrm{DM}}(\boldsymbol{\Omega}, g, \delta)+4 \pi g \int \widetilde{\rho}(\boldsymbol{x})^{2} d \boldsymbol{x}\right. \\
& \left.+\delta \int U(\boldsymbol{x}, \boldsymbol{y}) \widetilde{\rho}(\boldsymbol{x}) \widetilde{\rho}(\boldsymbol{y}) d \boldsymbol{x} d \boldsymbol{y}+C \operatorname{Tr}\left[\Gamma_{\Psi}^{(1)}(1-\widetilde{P})\right]\right)(1-o(1))
\end{aligned}
$$

for some constant $C>0$ (depending on $\Omega, g$ and $\delta$ ). Here $\Gamma_{\Psi}^{(1)}$ denotes the one-particle reduced density matrix of $\Psi$.

LEMMA 4. If $R \gg O\left(N^{-7 / 17}\right)$ and $o(1) \geq \varepsilon \gg O\left(N^{-2 / 17}\right)$ as $N \rightarrow \infty$, then

$$
\sum_{j=1}^{N} E_{j}^{(2)} \geq-N\left(4 \pi g \int \widetilde{\rho}(\boldsymbol{x})^{2} d \boldsymbol{x}+\delta \int U(\boldsymbol{x}, \boldsymbol{y}) \widetilde{\rho}(\boldsymbol{x}) \widetilde{\rho}(\boldsymbol{y}) d \boldsymbol{x} d \boldsymbol{y}\right)(1+o(1))
$$

Before proving these two Lemmas, let us show that they lead to Theorem 2 Inserting the lower bounds to $\sum_{j} E_{j}^{(1)}$ and $\sum_{j} E_{j}^{(2)}$ in (4.24) we obtain, for an appropriate choice of $R$ and $\varepsilon$,

$$
\begin{aligned}
\frac{1}{N}\langle\Psi| H_{N, \boldsymbol{\Omega}, a}+ & \frac{2 \delta}{N} \sum_{i<j} U\left(\boldsymbol{x}_{i}, \boldsymbol{x}_{j}\right)|\Psi\rangle \\
& \geq\left[\widetilde{E}^{\mathrm{DM}}(\boldsymbol{\Omega}, g, \delta)+C \operatorname{Tr}\left[\Gamma_{N}^{(1)}(1-\widetilde{P})\right]\right](1-o(1))
\end{aligned}
$$

as $N \rightarrow \infty$, if $N a \rightarrow g$. Together with the upper bound (4.21) this implies that

$$
\lim _{N \rightarrow \infty} \frac{1}{N} E^{\mathrm{QM}}\left(N, \Omega, g N^{-1}, \delta\right)=\widetilde{E}^{\mathrm{DM}}(\Omega, g, \delta)
$$

for all values of $\Omega, g$ and $\delta$ small, uniformly in $g$ on compact intervals, and also that

$$
\lim _{N \rightarrow \infty} \operatorname{Tr}\left[\Gamma_{N}^{(1)}(1-\widetilde{P})\right]=0
$$

Eq. (4.31) for $\delta=0$ proves (3.3). Moreover, since $\widetilde{E}^{\mathrm{DM}}$ is differentiable in $\delta$ at $\delta=0$, with derivative given in (4.16), we also infer that (see [18] for 
details)

$$
\lim _{N \rightarrow \infty} \int_{\mathbb{R}^{6}} U(\boldsymbol{x}, \boldsymbol{y}) \rho_{N}^{(2)}(\boldsymbol{x}, \boldsymbol{y}) d \boldsymbol{x} d \boldsymbol{y}=\int_{\mathbb{R}^{6}} U(\boldsymbol{x}, \boldsymbol{y}) \rho_{\boldsymbol{\Omega}, g}^{\mathrm{DM}}(\boldsymbol{x}) \rho_{\boldsymbol{\Omega}, g}^{\mathrm{DM}}(\boldsymbol{y}) d \boldsymbol{x} d \boldsymbol{y}
$$

where $\rho_{N}^{(2)}$ is the density of the reduced two-particle density matrix $\Gamma_{N}^{(2)}$ of an approximate ground state at $\delta=0$. This is true for all symmetric $U \in C_{0}^{\infty}\left(\mathbb{R}^{3} \times \mathbb{R}^{3}\right)$, i.e., $\rho_{N}^{(2)}(\boldsymbol{x}, \boldsymbol{y})$, being itself symmetric, converges to $\rho_{\boldsymbol{\Omega}, g}^{\mathrm{DM}}(\boldsymbol{x}) \rho_{\boldsymbol{\Omega}, g}^{\mathrm{DM}}(\boldsymbol{y})$ in the sense of distributions.

To show the convergence (3.4) of the density matrices, not only their densities, we proceed as follows. From (4.32) at $\delta=0$ we infer that

$$
\lim _{N \rightarrow \infty} \operatorname{Tr}\left[\Gamma_{N}^{(2)} P^{\mathrm{DM}} \otimes P^{\mathrm{DM}}\right]=1
$$

where $P^{\mathrm{DM}}$ is the projector onto the ground states of $\widetilde{H}$ with $\delta=0$, which is the operator appearing in the linear functional (2.5). Note that $P^{\mathrm{DM}}$ is finite dimensional, and $P^{\mathrm{DM}} \gamma_{\boldsymbol{\Omega}, g}^{\mathrm{DM}} P^{\mathrm{DM}}=\gamma_{\boldsymbol{\Omega}, g}^{\mathrm{DM}}$ by Prop. 10 From (4.34) we infer that there exists a subsequence of $\Gamma_{N}^{(2)}$ that converges to some $\Gamma$ in trace class norm, with $P^{\mathrm{DM}} \otimes P^{\mathrm{DM}} \Gamma P^{\mathrm{DM}} \otimes P^{\mathrm{DM}}=\Gamma$.

We want to show that the convergence (4.33) necessarily implies that $\Gamma=\gamma_{\boldsymbol{\Omega}, g}^{\mathrm{DM}} \otimes \gamma_{\boldsymbol{\Omega}, g}^{\mathrm{DM}}$. To do this, we have to take a closer look at the ground states of $\widetilde{H}$ for $\delta=0$. It is clear that they can be taken to be eigenfunctions of $\boldsymbol{\Omega} \cdot \boldsymbol{L}$, and that there is at most one ground state for any given eigenvalue of $\boldsymbol{\Omega} \cdot \boldsymbol{L}$. For eigenvalue $m \in \mathbb{Z}$, the ground state wave function can be written, in cylindrical coordinates $\boldsymbol{x}=(r, \varphi, z)$, as

$$
f(r, z) e^{i m \varphi},
$$

with $f(r, z)>0$ for all $r>0$ and all $z$, and $f(r, z) r^{-|m|}$ bounded (see [1] for details). Note that certainly $m \geq 0$ for any ground state. Expanding $\Gamma$ in terms of these eigenfunctions, and using the fact that because of (4.33) its density is necessarily given by $\rho_{\boldsymbol{\Omega}, g}^{\mathrm{DM}}(\boldsymbol{x}) \rho_{\boldsymbol{\Omega}, g}^{\mathrm{DM}}\left(\boldsymbol{x}^{\prime}\right)$, we obtain the equation

$$
\rho_{\boldsymbol{\Omega}, g}^{\mathrm{DM}}(\boldsymbol{x}) \rho_{\boldsymbol{\Omega}, g}^{\mathrm{DM}}\left(\boldsymbol{x}^{\prime}\right)=\sum_{j, k, l, m} a_{j k l m} f_{j}(r, z) f_{k}\left(r^{\prime}, z^{\prime}\right) f_{l}(r, z) f_{m}\left(r^{\prime}, z^{\prime}\right) e^{i(j-l) \varphi} e^{i(k-m) \varphi^{\prime}}
$$

for some coefficients $a_{j k l m}$ that determine $\Gamma$. This sum is finite, and since the left side does not depend on $\varphi$ and $\varphi^{\prime}$, we can infer that only terms with 
$j=l$ and $k=m$ contribute, i.e., $a_{j k l m}=0$ for $j \neq l$ or $k \neq m$. Here we have also used that terms with the same $j-l$, but different $j$ and $l$ can not cancel, because of the different asymptotics as $r \rightarrow 0$, namely $r^{j+l}$. By the same reasoning, we have

$$
\rho_{\boldsymbol{\Omega}, g}^{\mathrm{DM}}(\boldsymbol{x})=\sum_{j} \lambda_{j}\left|f_{j}(r, z)\right|^{2}
$$

for some $0 \leq \lambda_{j} \leq 1$. Using again the knowledge of the $r \rightarrow 0$ asymptotics of the $f_{j}$, we infer that $a_{j k j k}=\lambda_{j} \lambda_{k}$, and therefore $\Gamma=\gamma_{\boldsymbol{\Omega}, g}^{\mathrm{DM}} \otimes \gamma_{\boldsymbol{\Omega}, g}^{\mathrm{DM}}$. This proves (3.4) for $n=2$.

It remains to prove Lemmas 3 and 4 ,

Proof of Lemma 3. For fixed $\boldsymbol{X}_{j}$ define $f_{j}$ by

$$
f_{j}\left(\boldsymbol{x}_{j}\right)=\Psi\left(\boldsymbol{x}_{1}, \ldots, \boldsymbol{x}_{j}, \ldots, \boldsymbol{x}_{N}\right)
$$

and let

$$
W(\boldsymbol{x})=V(\boldsymbol{x})-\frac{|\boldsymbol{\Omega}|^{2}}{4} r^{2}+8 \pi g \widetilde{\rho}(\boldsymbol{x})+2 \delta \int U(\boldsymbol{x}, \boldsymbol{y}) \widetilde{\rho}(\boldsymbol{y}) d \boldsymbol{y} .
$$

We have

$$
E_{j}^{(1)} \geq(1-\varepsilon) \int_{\mathbb{R}^{3(N-1)}} d \boldsymbol{X}_{j} F_{j}-\frac{\varepsilon}{1-\varepsilon}\left\|[W]_{-}\right\|_{\infty}
$$

with

$$
F_{j}=\int_{\mathcal{O}_{j}}\left|(i \boldsymbol{\nabla}+\boldsymbol{A}) f_{j}\right|^{2}+\frac{\varepsilon}{2} \int_{\mathbb{R}^{3}}\left|(i \boldsymbol{\nabla}+\boldsymbol{A}) f_{j}\right|^{2}+\int_{\mathbb{R}^{3}} W\left|f_{j}\right|^{2} .
$$

The result now follows by applying Lemma 1 to $F_{j}$, noting that $\left|\mathcal{O}_{j}^{c}\right| \leq$ $N \frac{4 \pi}{3} R^{3}=o(1)$ and that

$$
\sum_{j=1}^{N} \int_{\mathbb{R}^{3(N-1)}} d \boldsymbol{X}_{j}\left\|f_{j}-P f_{j}\right\|_{2}^{2}=N \operatorname{Tr}\left[\Gamma_{\Psi}^{(1)}(1-\widetilde{P})\right]
$$

(compare with (4.14) and (4.15)). The constant $C$ is then given by the spectral gap of $\widetilde{H}$ above its ground state energy. Note that the last term in (4.40) is finite, by our assumption (1.2) on $V$ and the fact that $U$ is bounded and $\widetilde{\rho} \in L^{1}\left(\mathbb{R}^{3}\right)$. 
Proof of Lemma 4. To obtain a lower bound, we can use the box method, as in [16, 7]. More precisely, we divide $\mathbb{R}^{3}$ into boxes of side length $L$, labeled by $\alpha$, and distribute our $N$ particles over these boxes. Taking Neumann boundary conditions in each box and minimizing the energy with respect to all distributions of the particles, this can only lower the energy.

Let

$$
W_{\alpha}=\sup _{\boldsymbol{x} \in \alpha}\left[4 \pi g \widetilde{\rho}(\boldsymbol{x})+\delta \int_{\mathbb{R}^{3}} U(\boldsymbol{x}, \boldsymbol{y}) \widetilde{\rho}(\boldsymbol{y}) d \boldsymbol{y}\right]
$$

and

$$
U_{\alpha \beta}=\inf _{\boldsymbol{x} \in \alpha, \boldsymbol{y} \in \beta} U(\boldsymbol{x}, \boldsymbol{y}) .
$$

Using the diamagnetic inequality in the two terms in (4.26) containing $\boldsymbol{A}$, we can set $\boldsymbol{A}=\mathbf{0}$ for a lower bound. With $E_{\varepsilon}(n, L)$ defined as in Lemma 2 we thus obtain

$$
\begin{aligned}
& \sum_{j=1}^{N} E_{j}^{(2)} \geq \\
& \quad \inf _{\left\{n_{\alpha}\right\}}\left\{\sum_{\alpha}\left(E_{\varepsilon}\left(n_{\alpha}, L\right)-2 W_{\alpha} n_{\alpha}\right)+\frac{\delta}{N} \sum_{\alpha, \beta} U_{\alpha \beta} n_{\alpha} n_{\beta}-\frac{\delta}{N} \sum_{\alpha} U_{\alpha \alpha} n_{\alpha}\right\},
\end{aligned}
$$

where the infimum is taken over all distribution of the $N$ particles into the boxes $\alpha$. Each box contains $n_{\alpha}$ particles, and $\sum_{\alpha} n_{\alpha}=N$. The last term in (4.45) is due to the fact that there are only $\frac{1}{2} n(n-1)$ pairs of particles in the same box. It can easily be estimated by $\frac{1}{2} \delta\|U\|_{\infty}$, which is negligible compared to the other terms of order $N$.

Let $\bar{n}_{\alpha}$ be a minimizing configuration of the $n_{\alpha}$ 's in (4.45). Let $Y=$ $a^{3} N / L^{3}$, and let $\Lambda_{\eta}$ denote the collection of those boxes $\alpha$ where $\bar{n}_{\alpha} \geq \eta N L^{3}$. If we choose $\varepsilon \geq Y^{1 / 17}$ and $R \geq a\left(a^{3} \eta N\right)^{-5 / 17}$ (compare with the conditions stated in Lemma 2) we can use (4.11) to estimate, for $\alpha \in \Lambda_{\eta}$,

$$
E_{\varepsilon}\left(\bar{n}_{\alpha}, L\right) \geq 4 \pi a \frac{\bar{n}_{\alpha}^{2}}{L^{3}}\left(1-C Y^{1 / 17}\right)(1-\varepsilon)
$$

where we estimated $\bar{n}_{\alpha}$ by $N$ in the error term. For $\alpha \notin \Lambda_{\eta}$ we simply use 
$E_{\varepsilon}\left(\bar{n}_{\alpha}, L\right) \geq 0$. This gives

(4.45)

$$
\begin{aligned}
\geq \sum_{\alpha \in \Lambda_{\eta}, \beta \in \Lambda_{\eta}} \bar{n}_{\alpha} \bar{n}_{\beta} & {\left[\delta_{\alpha \beta} \frac{4 \pi g}{N L^{3}}\left(1-C Y^{1 / 17}\right)(1-\widehat{\varepsilon})+\frac{\delta}{N} U_{\alpha \beta}\right]-\sum_{\alpha \in \Lambda_{\eta}} 2 W_{\alpha} \bar{n}_{\alpha} } \\
& -2 \eta N L^{3}\left(\sum_{\alpha} W_{\alpha}+\delta \sup _{\beta} \sum_{\alpha} U_{\alpha \beta}\right)-\frac{1}{2} \delta\|U\|_{\infty} \cdot
\end{aligned}
$$

Here $\widehat{\varepsilon}=1-(1-\varepsilon) N a / g=o(1)$ for large $N$.

In the following, we will choose $L=o(1)$ and $\eta=o(1)$ as $N \rightarrow \infty$. The term in brackets in the last line in (4.47) can then be bounded by const. $L^{-3}$, which is, when multiplied by $\eta N L^{3}$, of lower order than $N$ and therefore negligible compared to the main terms of order $N$.

Consider now the first two terms in (4.47). If $\delta$ is small enough (independent of $N$ and $L$ ), the term in square brackets defines a positive matrix $B_{\alpha \beta}$ for $N$ large enough. Moreover, denoting $\rho_{\alpha}=\sup _{\boldsymbol{x} \in \alpha} \widetilde{\rho}(\boldsymbol{x})$,

$$
W_{\alpha}=N L^{3} \sum_{\beta} B_{\alpha \beta} \rho_{\beta}+\ell_{\alpha}
$$

where $\ell_{\alpha}=\ell_{\alpha}^{(1)}+\ell_{\alpha}^{(2)}$, with

$$
\ell_{\alpha}^{(1)}=4 \pi g\left(C Y^{1 / 17}+\widehat{\varepsilon}-\widehat{\varepsilon} C Y^{1 / 17}\right) \rho_{\alpha}
$$

and

$$
\ell_{\alpha}^{(2)}=\delta\left(\sup _{\boldsymbol{x} \in \alpha} \int U(\boldsymbol{x}, \boldsymbol{y}) \widetilde{\rho}(\boldsymbol{y}) d \boldsymbol{y}-L^{3} \sum_{\beta} U_{\alpha \beta} \rho_{\beta}\right) .
$$

In the following we denote by $\boldsymbol{B}$ the matrix with coefficients $B_{\alpha \beta}$, and likewise by $\boldsymbol{W}, \boldsymbol{\rho}, \boldsymbol{\ell}$ the vectors with components $W_{\alpha}, \rho_{\alpha}$ and $\ell_{\alpha}$, respectively. Let also $\boldsymbol{n}$ denote the vector with entries $\bar{n}_{\alpha}$ for $\alpha \in \Lambda_{\eta}$ and 0 for $\alpha \notin \Lambda_{\eta}$. With these notations we can rewrite the first two terms on the right side of (4.47) as

$$
\begin{aligned}
& \langle\boldsymbol{n}|\boldsymbol{B}| \boldsymbol{n}\rangle-2\langle\boldsymbol{W} \mid \boldsymbol{n}\rangle \\
& \quad=\left\langle\boldsymbol{n}-N L^{3} \boldsymbol{\rho}|\boldsymbol{B}| \boldsymbol{n}-N L^{3} \boldsymbol{\rho}\right\rangle-\left\langle N L^{3} \boldsymbol{\rho}|\boldsymbol{B}| N L^{3} \boldsymbol{\rho}\right\rangle-2\langle\ell \mid \boldsymbol{n}\rangle .
\end{aligned}
$$


Because of positivity of $\boldsymbol{B}$, this expression is bounded below by

$$
-\left\langle N L^{3} \boldsymbol{\rho}|\boldsymbol{B}| N L^{3} \boldsymbol{\rho}\right\rangle-2 N\|\boldsymbol{\ell}\|_{\infty} .
$$

Now, if we choose $L=o(1)$ as $N \rightarrow \infty$, we have

$$
\left(N L^{3}\right)^{2}\langle\boldsymbol{\rho}|\boldsymbol{B}| \boldsymbol{\rho}\rangle=N\left(4 \pi g \int \widetilde{\rho}(\boldsymbol{x})^{2}+\delta \int U(\boldsymbol{x}, \boldsymbol{y}) \widetilde{\rho}(\boldsymbol{x}) \widetilde{\rho}(\boldsymbol{y})\right)(1+o(1))
$$

because the sums are Riemann sums for the corresponding integrals. Moreover, $\|\ell\|_{\infty} \leq o(1)$, which is obvious for (4.49), since $\rho_{\alpha}$ is bounded, and also for (4.50) by the same Riemann sum argument as above. This proves the Lemma.

This finishes the proof of (3.4) for $n=2$. The general case $n \geq 1$ follows in the same manner, perturbing the Hamiltonian (1.4) by

$$
\delta \frac{n !}{N^{n-1}} \sum_{1 \leq i_{1}<\cdots<i_{n} \leq N} U\left(\boldsymbol{x}_{i_{1}}, \ldots, \boldsymbol{x}_{i_{n}}\right)
$$

where $U \in C_{0}^{\infty}\left(\mathbb{R}^{3 n}\right)$ is a realvalued symmetric function of $n$ variables, and also perturbing the DM functional by a term

$$
\delta \int_{\mathbb{R}^{3 n}} U\left(\boldsymbol{x}_{1}, \ldots, \boldsymbol{x}_{n}\right) \rho_{\gamma}\left(\boldsymbol{x}_{1}\right) \cdots \rho_{\gamma}\left(\boldsymbol{x}_{n}\right)
$$

We will only sketch the proof here, pointing out the differences to the case $n=2$. Following the same steps as in the proof of Lemma 4 above, one sees that minimizing over particle numbers in different boxes is, after passing to the limit $N \rightarrow \infty$, effectively equivalent to minimizing a functional

$$
\rho \mapsto \int_{\mathbb{R}^{3}}\left[4 \pi g \rho(\boldsymbol{x})^{2}-W(\boldsymbol{x}) \rho(\boldsymbol{x})\right]+\delta \int_{\mathbb{R}^{3 n}} U\left(\boldsymbol{x}_{1}, \ldots, \boldsymbol{x}_{n}\right) \rho\left(\boldsymbol{x}_{1}\right) \cdots \rho\left(\boldsymbol{x}_{n}\right)
$$

over functions $\rho \in L^{1}\left(\mathbb{R}^{3}\right)$ with $\rho \geq 0$ and $\int \rho=1$. Here

$$
W(\boldsymbol{x})=8 \pi g \widetilde{\rho}(\boldsymbol{x})+n \delta \int_{\mathbb{R}^{3(n-1)}} U\left(\boldsymbol{x}, \boldsymbol{x}_{2}, \ldots, \boldsymbol{x}_{n}\right) \widetilde{\rho}\left(\boldsymbol{x}_{2}\right) \cdots \widetilde{\rho}\left(\boldsymbol{x}_{n}\right),
$$

and $\widetilde{\rho}$ is the density of a minimizer of the modified DM functional with additional term (4.55), with $\int \widetilde{\rho}=1$. To obtain the analogue of Lemma 4 we 
have to to show that the minimizer of (4.56) is given by $\widetilde{\rho}$, at least for $\delta$ small enough (compare with (4.51), which is just a discretized version of (4.56) for $n=2)$. Compared to the case $n=2$ there is an additional difficulty here since the functional (4.56) is not necessarily convex. (The convexity in the case $n=2$ was used in the form of positivity of $\boldsymbol{B}$.)

To show that, for small enough $\delta$, the minimizer of the functional (4.56) is given by $\widetilde{\rho}$, we rewrite (4.56) as

$$
-4 \pi g \int \widetilde{\rho}(\boldsymbol{x})^{2}-(n-1) \delta \int U\left(\boldsymbol{x}_{1}, \ldots, \boldsymbol{x}_{n}\right) \widetilde{\rho}\left(\boldsymbol{x}_{1}\right) \cdots \widetilde{\rho}\left(\boldsymbol{x}_{n}\right)+\mathcal{F}[\rho],
$$

with

$$
\begin{aligned}
\mathcal{F}[\rho] & =4 \pi g \int(\rho-\widetilde{\rho})^{2} \\
& +\sum_{i=1}^{n} c_{i} \int_{\mathbb{R}^{3 i}} U^{(i)}\left(\boldsymbol{x}_{1}, \ldots, \boldsymbol{x}_{i}\right)\left(\rho\left(\boldsymbol{x}_{1}\right)-\widetilde{\rho}\left(\boldsymbol{x}_{1}\right)\right) \cdots\left(\rho\left(\boldsymbol{x}_{i}\right)-\widetilde{\rho}\left(\boldsymbol{x}_{i}\right)\right)
\end{aligned}
$$

where

$$
U^{(i)}\left(\boldsymbol{x}_{i}, \ldots, \boldsymbol{x}_{i}\right)=\int_{\mathbb{R}^{3(n-i)}} U\left(\boldsymbol{x}_{1}, \ldots, \boldsymbol{x}_{n}\right) \widetilde{\rho}\left(\boldsymbol{x}_{i+1}\right) \cdots \widetilde{\rho}\left(\boldsymbol{x}_{n}\right) d \boldsymbol{x}_{i+1} \cdots d \boldsymbol{x}_{n}
$$

and $c_{i} \in \mathbb{N}$ are appropriate integer coefficients. Now $\mathcal{F}[\widetilde{\rho}]=0$, so any minimizer $\rho$ must fulfill $\mathcal{F}[\rho] \leq 0$. Since $\|\rho-\widetilde{\rho}\|_{1} \leq 2$ by assumption, we have $\mathcal{F}[\rho] \geq 4 \pi g\|\rho-\widetilde{\rho}\|_{2}^{2}-$ const. $\delta\|U\|_{\infty}$, and hence

$$
\|\rho-\widetilde{\rho}\|_{2}^{2} \leq \text { const. } \delta
$$

for a minimizer $\rho$. On the other hand,

$$
\mathcal{F}[\rho] \geq 4 \pi g\|\rho-\widetilde{\rho}\|_{2}^{2}-\text { const. } \delta\|U\|_{2}\left(\|\rho-\widetilde{\rho}\|_{2}^{2}+\|\rho-\widetilde{\rho}\|_{2}^{n}\right),
$$

which is, because of (4.61), positive for $\delta$ small enough, and zero only for $\rho=\widetilde{\rho}$. Therefore $\widetilde{\rho}$ is the unique minimizer of (4.56).

The convergence of the energies for a small interval of $\delta$ 's around zero implies again the convergence of the $n$-particle densities at $\delta=0$, and by analogous arguments as in the case $n=2$ one can show the convergence of the density matrices. The details are left to the reader.

This finishes the proof of Theorem [2] 


\subsection{Proof of Theorem 3}

We start by computing an upper bound to $E_{\text {bose }}^{\mathrm{QM}}$. Note that we can not use (4.18) as a trial state, for two reasons. First of all, $F$ is not a symmetric function and, secondly, the tensor product of one-particle density matrices is not a bosonic density matrix, if their rank is bigger than one. The first problem turns out not be serious (see below), but the second is.

Instead of (4.18), we use as a trial function

$$
\Psi\left(\boldsymbol{x}_{1}, \ldots, \boldsymbol{x}_{N}\right)=F\left(\boldsymbol{x}_{1}, \ldots, \boldsymbol{x}_{N}\right) \prod_{i=1}^{N} \phi^{\mathrm{GP}}\left(\boldsymbol{x}_{i}\right)
$$

where $\phi^{\mathrm{GP}}$ is a minimizer of the GP functional, and $F$ is given by the Dyson wave function (4.19). Note that $F$ is not a symmetric function, but we claim that (4.63) nevertheless gives an upper bound to $E_{\text {bose }}^{\mathrm{QM}}$. More precisely, we will show that any trial function of the form (4.63) with $F$ realvalued but not necessarily symmetric gives an upper bound to $E_{\text {bose }}^{\mathrm{QM}}$. To see this, we write, for $\Psi$ given in (4.63),

$$
\begin{gathered}
\left\langle\Psi\left|H_{N, \boldsymbol{\Omega}, a}\right| \Psi\right\rangle=\mu_{\boldsymbol{\Omega}, g}^{\mathrm{GP}}\langle\Psi \mid \Psi\rangle+\sum_{j=1}^{N} \int_{\mathbb{R}^{3 N}} \prod_{k=1}^{N}\left|\phi^{\mathrm{GP}}\left(\boldsymbol{x}_{k}\right)\right|^{2} d \boldsymbol{x}_{k}\left[\left|\nabla_{j} F\right|^{2}\right. \\
\left.+\left(\frac{1}{2} \sum_{i=1, i \neq j}^{N} \frac{1}{a^{2}} v\left(\left(\boldsymbol{x}_{i}-\boldsymbol{x}_{j}\right) / a\right)-8 \pi g\left|\phi^{\mathrm{GP}}\left(\boldsymbol{x}_{j}\right)\right|^{2}\right)|F|^{2}\right] \prod_{k=1}^{N}\left|\phi^{\mathrm{GP}}\left(\boldsymbol{x}_{k}\right)\right|^{2} d \boldsymbol{x}_{k}
\end{gathered}
$$

where we used partial integration, the GP equation (2.6), and the fact that $F$ is real by assumption. The reality of $F$ has the effect the both the crossterm in the partial integration and the term with $\boldsymbol{L}$ acting on $F$ vanish. The infimum of (4.64) over all $F$ is attained for a positive $F$, and therefore we can proceed as in [20, p. 15] to show that the infimum of (4.64) over all $F$ is the same as the infimum over symmetric $F$.

It is therefore legitimate to use (4.63) as a trial function for the bosonic ground state problem. The calculation of the expectation value of $H_{N, \boldsymbol{\Omega}, a}$ follows again along the same lines as in [7, with the result that

$$
E_{\text {bose }}^{\mathrm{QM}}\left(N, \boldsymbol{\Omega}, g N^{-1}\right) \leq N E^{\mathrm{GP}}(\boldsymbol{\Omega}, g)\left(1+O\left(N^{-2 / 3}\right)\right) .
$$

Letting $N \rightarrow \infty$ this proves (3.5). 
From (3.5) it follows immediately that

$$
\limsup _{N \rightarrow \infty} \frac{1}{N} E_{\text {bose }}^{\mathrm{QM}}\left(N, \boldsymbol{\Omega}, g N^{-1}\right)>E^{\mathrm{DM}}(\boldsymbol{\Omega}, g)
$$

implies (3.7). Since (3.6) clearly implies (4.66), it remains to show that (3.6) follows from (3.7). To see this, note that $E^{\mathrm{GP}}(\boldsymbol{\Omega}, g)>E^{\mathrm{DM}}(\boldsymbol{\Omega}, g)$ implies that the unique minimizer $\gamma_{\boldsymbol{\Omega}, g}^{\mathrm{DM}}$ of $\mathcal{E}_{\boldsymbol{\Omega}, g}^{\mathrm{DM}}$ has at least rank 2. Therefore $\gamma_{\boldsymbol{\Omega}, g}^{\mathrm{DM}} \otimes \gamma_{\boldsymbol{\Omega}, g}^{\mathrm{DM}}$ is not a bosonic two-particle density matrix, it has both symmetric and antisymmetric eigenfunctions. Since the space of bosonic two-particle density matrices is closed under the trace norm, (3.4) cannot be true for the bosonic ground state in this case. Theorem 2 implies that the bosonic ground state is not an approximate ground state for the unrestricted problem, and hence (3.6) holds. This proves Theorem 3, The uniformity of the limit (3.4) (see the definition of approximate ground state in (3.1)) implies uniformity in (3.6), as stated in (3.8) in Remark 1.

We conclude this section with a proof of the assertion (3.11) made in Remark 2 that the Gibbs states (3.10) are approximate ground states. Let $T=1 / \beta$. We start by estimating the free energy

$$
F(T, N)=-\frac{1}{\beta} \ln \operatorname{Tr}\left[P_{\text {bose }} \exp \left(-\beta H_{N}\right)\right]
$$

For simplicity of notation we suppress the dependence on $\Omega$ and $a$ from now on. It is always understood that $a=g N^{-1}$. The function $F$ is monotone decreasing and concave in $T$. This implies immediately that $F(T, N) \leq$ $F(0, N)=E_{\text {bose }}^{\mathrm{QM}}(N)$. To obtain a lower bound, we proceed as follows. Let $n(e)$ denote the number of eigenvalues of $H_{N} \uparrow_{P_{\text {bose }} \mathcal{H}_{N}}$ that are smaller or equal to $e$. Then

$$
F(T, N)=-\frac{1}{\beta} \ln \left[\beta \int_{E_{\mathrm{bose}}^{\mathrm{QM}}(N)}^{\infty} n(e) \exp (-\beta e) d e\right]
$$

Since $v \geq 0, n(e)$ is bounded above by the number of eigenvalues of the non-interacting Hamiltonian $\sum_{i} H_{0}^{(i)} \uparrow_{P_{\text {bose }} \mathcal{H}_{N}}$ that are smaller or equal to $e$. This, in turn, can be bounded above by

$$
\operatorname{Tr}\left[P_{\text {bose }} \exp \left(\alpha e-\alpha \sum_{i} H_{0}^{(i)}\right)\right] \equiv \exp (\alpha e) C_{\alpha, N}
$$


for any $\alpha>0$. Choosing $\alpha<\beta$ we therefore obtain

$$
F(T, N) \geq \frac{\beta-\alpha}{\beta} E_{\text {bose }}^{\mathrm{QM}}(N)-\frac{1}{\beta} \ln \left(\frac{\beta C_{\alpha, N}}{\beta-\alpha}\right) .
$$

Now let $e_{i}, i \geq 0$, denote the ordered sequence of eigenvalues of $H_{0}$, including degenerate eigenvalues, and let $j_{0}$ denote the multiplicity of the lowest eigenvalue $e_{0}$. The expression $C_{\alpha, N}$ can easily be bounded above using the fact that any totally symmetric eigenfunction of $\sum_{i} H_{0}^{(i)}$ can be characterized by the occupation numbers $n_{j}$ of the eigenfunctions of $H_{0}$ corresponding to the eigenvalue $e_{j}$. Thus $C_{\alpha, N}$ can be expressed as

$$
\begin{aligned}
C_{\alpha, N} & =e^{-\alpha N e_{0}} \operatorname{Tr}\left[P_{\text {bose }} \exp \left(-\alpha \sum_{i=1}^{N}\left(H_{0}^{(i)}-e_{0}\right)\right)\right] \\
& =e^{-\alpha N e_{0}} \sum_{\left\{n_{j} \in \mathbb{N}_{0}\right\}_{j \geq 0}, \sum n_{j}=N} e^{-\alpha \sum n_{j}\left(e_{j}-e_{0}\right)},
\end{aligned}
$$

where we introduced the notation $\mathbb{N}_{0}=\mathbb{N} \cup\{0\}$. To bound this expression from above, we simply neglect the condition $\sum_{j \geq 0} n_{j}=N$. More precisely, we estimate

$$
\begin{aligned}
C_{\alpha, N} & \leq e^{-\alpha N e_{0}} \sum_{n_{0}=0}^{N} \cdots \sum_{n_{j_{0}-1}=0}^{N} \sum_{\left\{n_{j} \in \mathbb{N}_{0}\right\}_{j \geq j_{0}}} e^{-\alpha \sum n_{j}\left(e_{j}-e_{0}\right)} \\
& =(N+1)^{j_{0}} e^{-\alpha N e_{0}} \prod_{j=j_{0}}^{\infty} \frac{1}{1-e^{-\alpha\left(e_{j}-e_{0}\right)}} .
\end{aligned}
$$

Note that the last product is finite, and independent of $N$. Inserting the bound (4.72) in (4.70), letting $N \rightarrow \infty$ and afterwords $\alpha \rightarrow 0$, we obtain

$$
\lim _{N \rightarrow \infty} \frac{1}{N}\left(F(T, N)-E_{\text {bose }}^{\mathrm{QM}}(N)\right)=0 \text {. }
$$

To see that the same convergence holds for the energies

$$
E(T, N)=\frac{\operatorname{Tr}\left[P_{\text {bose }} H_{N} \exp \left(-\beta H_{N}\right)\right]}{\operatorname{Tr}\left[P_{\text {bose }} \exp \left(-\beta H_{N}\right)\right]},
$$

note that

$$
\frac{\partial F(T, N)}{\partial T}=\frac{1}{T}(F(T, N)-E(T, N)) .
$$

The concavity of $F(T, N)$ in $T$ and (4.73) imply that $N^{-1} \partial F / \partial T \rightarrow 0$ as $N \rightarrow \infty$, which proves the desired result. 


\subsection{Proof of Corollary 1}

The first statement on the impossibility of having a totally symmetric ground state follows immediately from Theorem 3. To estimate the ground state degeneracy, we proceed as follows. Since the Hamiltonian under consideration is symmetric with respect to exchange of two variables, any ground state belongs to a finite dimensional space of an irreducible representation of $S_{N}$, the permutation group of $N$ variables. These representations are conveniently labeled by Young tableaux. Given the ground state space of $H_{N, \boldsymbol{\Omega}, g N^{-1}}$ and one of the corresponding representations of $S_{N}$, let, for fixed $(|\boldsymbol{\Omega}|, g) \in \Xi$, $R_{N}$ and $C_{N}$ denote the length of the longest row and column of the Young tableaux, respectively. We will show that $C_{N}$ is bounded above independent of $N$, and $R_{N} / N<1-\varepsilon$ for some $\varepsilon>0$ and $N$ large enough. This will allow us to show that the dimension of the representation increases exponentially with $N$.

We first compute an upper bound on $C_{N}$. There exists a ground state of $H_{N, \Omega, g N^{-1}}$ which is antisymmetric in the first $C_{N}$ variables. Neglecting the interaction with the other particles, we therefore have

$$
E^{\mathrm{QM}}\left(N, \Omega, g N^{-1}\right) \geq \operatorname{Tr}\left[H_{0} P_{C_{N}}\right]+E^{\mathrm{QM}}\left(N-C_{N}, \Omega, g N^{-1}\right),
$$

where $P_{C}$ is the projection of $H_{0}$ onto the first $C$ eigenvalues. By analogous considerations as in the upper bound to $E^{\mathrm{QM}}$ (cf. also [7, Thm. III.2]) it is not difficult to show that

$$
E^{\mathrm{QM}}\left(N, \Omega, g N^{-1}\right)-E^{\mathrm{QM}}\left(N-C, \Omega, g N^{-1}\right) \leq \text { const. } C
$$

for fixed $g$ and $\boldsymbol{\Omega}$, independent of $N$. Since

$$
\lim _{C \rightarrow \infty} \frac{1}{C} \operatorname{Tr}\left[H_{0} P_{C}\right]=\infty
$$

because of our assumption that $V$ goes to infinity at infinity, this implies that $C_{N}$ has to stay bounded as $N \rightarrow \infty$.

We now derive an upper bound on $R_{N}$. Since there is a ground state of $H_{N, \Omega, g N^{-1}}$ which is symmetric in the first $R_{N}$ variables, we have, by analogous considerations as above,

$$
E^{\mathrm{QM}}\left(N, \Omega, g N^{-1}\right) \geq E_{\text {bose }}^{\mathrm{QM}}\left(R_{N}, \Omega, g N^{-1}\right)+\left(N-R_{N}\right) E_{0},
$$


where $E_{0}=\inf \operatorname{spec} H_{0}$. Let $\eta=\lim \sup _{N \rightarrow \infty} R_{N} / N$, and choose a subsequence such that $R_{N} / N \rightarrow \eta$ as $N \rightarrow \infty$. It follows from (4.79) and Theorem 2 that

$$
\left.\eta \leq\left(E^{\mathrm{DM}}(\Omega, g)-E_{0}\right)\left(\liminf _{N \rightarrow \infty} \frac{1}{R_{N}} E_{\mathrm{bose}}^{\mathrm{QM}}\left(R_{N}, \Omega, g R_{N}^{-1} R_{N} / N\right)\right)-E_{0}\right)^{-1}
$$

Since certainly $R_{N} \rightarrow \infty$ with $N$, we can use (3.8) to conclude that

$$
\left.\liminf _{N \rightarrow \infty} \frac{1}{R_{N}} E_{\text {bose }}^{\mathrm{QM}}\left(R_{N}, \Omega, g R_{N}^{-1} R_{N} / N\right)\right)>E^{\mathrm{DM}}(\Omega, g \eta)
$$

if $1-\eta$ is small enough such that $(|\Omega|, g \eta) \in \Xi$. This shows that $\eta<1$ for $(|\Omega|, g) \in \Xi$.

We have thus shown that, for some $\varepsilon>0, R_{N} \leq N(1-\varepsilon)$ and $C_{N} \leq$ $1 / \varepsilon$ for $N$ large enough. Therefore there are at least two rows of length $\geq \varepsilon^{2} N$ in the Young tableaux, and hence the dimension of the corresponding representation is at least the one of the tableaux consisting of two rows of length $\ell=\left[\varepsilon^{2} N\right]$ (here the square bracket denotes the integer part). This is given by

$$
\frac{(2 \ell) !}{\ell !(\ell+1) !} \sim \frac{4^{\ell}}{\ell^{3 / 2}} \quad \text { for large } \ell
$$

which proves the Corollary.

To prove the statement on the upper bound on the ground state degeneracy in Remark 3 after Cor. 1 we estimate, for $\theta(z)=1$ for $z \geq 1$ and $\theta(z)=0$ for $z<1$,

$$
\begin{aligned}
\mathcal{N}\left(N,|\boldsymbol{\Omega}|, g N^{-1}\right) & =\operatorname{Tr}\left[\theta\left(E^{\mathrm{QM}}\left(N, \boldsymbol{\Omega}, g N^{-1}\right)-H_{N, \boldsymbol{\Omega}, g N^{-1}}\right)\right] \\
& \leq \operatorname{Tr}\left[\theta\left(N\left(E^{\mathrm{DM}}(\boldsymbol{\Omega}, g)+\varepsilon\right)-\sum_{i=1}^{N} H_{0}^{(i)}\right)\right]
\end{aligned}
$$

for any $\varepsilon>0$ and $N$ large enough. Here we have used (3.3) and the fact that the interaction potential $v$ is positive by assumption. Estimating the step function by the exponential function, we therefore get, for any $\beta>0$,

$$
\begin{aligned}
\mathcal{N}\left(N,|\boldsymbol{\Omega}|, g N^{-1}\right) & \leq \operatorname{Tr}\left[\exp \left(\beta\left(N\left(E^{\mathrm{DM}}(\boldsymbol{\Omega}, g)+\varepsilon\right)-\sum_{i=1}^{N} H_{0}^{(i)}\right)\right)\right] \\
& =\operatorname{Tr}\left[\exp \left(\beta\left(E^{\mathrm{DM}}(\boldsymbol{\Omega}, g)+\varepsilon-H_{0}\right)\right)\right]^{N}
\end{aligned}
$$

which proves (3.13). 


\section{A Appendix: Proof of Theorem 1}

The strategy of the proof of Theorem 1 is similar to the one in the twodimensional case given in in [11. One shows the non-uniqueness of minimizers of the GP functional by showing that no eigenfunction of the angular momentum $\boldsymbol{\Omega} \cdot \boldsymbol{L}$ can be a minimizer, even though the GP functional is invariant under rotations around the $\Omega$ axis. This implies that the minimizer cannot be unique, since by rotating a minimizer one gets another one. Moreover, since the minimizer of the DM functional $\mathcal{E}_{\Omega, g}^{\mathrm{DM}}$ is unique by Prop. 1. this implies that it has to have at least rank 2 , and $E^{\mathrm{DM}}(\Omega, g)<E^{\mathrm{GP}}(\boldsymbol{\Omega}, g)$, as stated in Theorem 1.

Thus all we have to show is that an eigenfunction of $\boldsymbol{\Omega} \cdot \boldsymbol{L}$ cannot minimize $\mathcal{E}_{\boldsymbol{\Omega}, g}^{\mathrm{GP}}$. To do this, we first have to consider the properties of minimizers in the subspaces of angular momentum eigenfunctions. Except for Lemma [6] all the considerations below follow the same line as in the two-dimensional case. Lemma 6, however, is the main new ingredient to extend the results to three dimensions. While Lemmas 56 7 hold for general $V$ 's, our assumptions (2.8) and (2.9) on the external potential $V$ will become important in the proof of Theorem 1 below, where they are used in order to get explicit estimates on the various quantities appearing in Lemmas 5 , 7.

Let $f_{n}$ denote the minimizer of the GP functional restricted to functions $\phi(\boldsymbol{x})=f(r, z) e^{i n \varphi}$, i.e., to functions with angular momentum $\boldsymbol{\Omega} \cdot \boldsymbol{L}=n$. More precisely, $f_{n}$ minimizes

$$
f \mapsto \int_{0}^{\infty} d^{2} r \int_{-\infty}^{\infty} d z f^{*}\left(-\Delta_{r} f-\frac{\partial^{2} f}{\partial z^{2}}+\frac{n^{2}}{r^{2}} f+V f+4 \pi g|f|^{2} f\right)
$$

under the normalization condition $\int|f(r, z)|^{2} d^{2} r d z=1$, where we denoted $d^{2} r=2 \pi r d r$, and $\Delta_{r}=\partial^{2} / \partial r^{2}+r^{-1} \partial / \partial r$. In the following, it will be convenient to study this functional for all $n \in \mathbb{R}_{+}$, not only for integers. Using standard methods one can show that for each $n \geq 0$ and $g \geq 0$ there exists a unique minimizer of (A.1). Let $E_{n}(g)$ denote the corresponding minimum of (A.1), and let

$$
\widetilde{\mu}_{n}=E_{n}(g)+4 \pi g \int\left|f_{n}(r, z)\right|^{4} d^{2} r d z .
$$

Note that $\widetilde{\mu}_{n}$ depends on $g$ besides $n$.

We need the following estimates on the minimizers $f_{n}$. 
LEMMA 5. The minimizer $f_{n}$ is a non-negative, bounded function, with

$$
\left\|f_{n}\right\|_{\infty} \leq \frac{\widetilde{\mu}_{n}}{8 \pi g}
$$

Proof. As in [11, Lemma 1] this follows from the maximum principle, applied to the variational equation for $f_{n}$, which reads

$$
-\Delta_{r} f_{n}-\frac{\partial^{2}}{\partial z^{2}} f_{n}+\frac{n^{2}}{r^{2}} f_{n}+V f_{n}=\left(\widetilde{\mu}_{n}-8 \pi g\left|f_{n}\right|^{2}\right) f_{n} .
$$

Recall that $V \geq 0$ by assumption.

LEMMA 6. Let $j_{n}(r)=\int_{-\infty}^{\infty} d z\left|f_{n}(r, z)\right|^{2}$. Choose $R>0$ large enough such that

$$
\delta_{n}(R) \equiv \inf _{|z| \geq R} V(\boldsymbol{x})-\widetilde{\mu}_{n}>0
$$

which is always possible by our assumption that $V$ goes to infinity at infinity. Then

$$
\left\|j_{n}\right\|_{\infty} \leq\left\|f_{n}\right\|_{\infty}^{2}\left(4 R+\text { const. } \frac{1}{R \delta_{n}(R)}\right)
$$

Here $\|\cdot\|$ has to be understood as the supremum norm on $\mathbb{R}^{2}$ and $\mathbb{R}^{3}$, respectively.

Proof. Let $\eta \in C^{\infty}(\mathbb{R})$ be such that $0 \leq \eta \leq 1, \eta(z)=1$ for $|z| \geq 2$ and $\eta(z)=0$ for $|z| \leq 1$. Then

$$
j_{n}(r)=\int_{-\infty}^{\infty} d z\left|f_{n}(r, z)\right|^{2}((1-\eta(z / R))+\eta(z / R)) \leq 4 R\left\|f_{n}\right\|_{\infty}^{2}+\chi(r)
$$

where we denoted

$$
\chi(r)=\int d z\left|f_{n}(r, z)\right|^{2} \eta(z / R)
$$

Using the variational equation for $f_{n}$ and neglecting positive terms, it is straightforward to derive the inequality

$$
-\Delta_{r} \chi \leq 2 \int d z \eta(z / R)\left(\left|f_{n}\right|^{2}\left(\widetilde{\mu}_{n}-V(r, z)\right)+f_{n} \frac{\partial^{2}}{\partial z^{2}} f_{n}\right) .
$$


Partial integration yields the estimate

$$
2 \int d z \eta(z / R)\left(f_{n} \frac{\partial^{2}}{\partial z^{2}} f_{n}\right) \leq \frac{1}{R^{2}} \int d z\left|f_{n}\right|^{2} \eta^{\prime \prime}(z / R) \leq \frac{\text { const. }}{R}\|f\|_{\infty}^{2} \equiv \varepsilon .
$$

Using (A.5) and the support properties of $\eta$ we therefore get

$$
-\Delta_{r} \chi \leq \varepsilon-2 \delta_{n}(R) \chi
$$

It follows from the maximum principle that $\chi(r) \leq \varepsilon /\left(2 \delta_{n}(R)\right)$, which proves our claim.

LEMMA 7. With $j_{n}$ given in Lemma 6, $r^{-2 n} j_{n}(r)$ is a bounded function, with

$$
\left\|r^{-2 n} j_{n}\right\|_{\infty} \leq\left(2 c_{n}^{2} \widetilde{\mu}_{n}\right)^{n}\left\|j_{n}\right\|_{\infty}
$$

where $c_{n}$ is given by

$$
\begin{aligned}
& c_{n}=\left(\frac{2^{-n}\left(\frac{2-n}{n}\right)^{n / 2} \pi \operatorname{Csc}\left(\frac{n \pi}{2}\right)}{(2-n) \Gamma(n)}\right)^{1 / n} \quad \text { for } n \leq 1 \\
& c_{n}=\frac{\sqrt{\pi}}{n} \frac{\Gamma\left(n+\frac{1}{2}\right)}{\Gamma(n)} \quad \text { for } n \geq 1 .
\end{aligned}
$$

Proof. The proof of this Lemma is similar to the corresponding Lemma 2 in [1]. Let $h(r)=r^{-2 n} \chi(r)$. Using the variational equation for $f_{n}$ and the fact that $V$ is positive by assumption, one derives the differential inequality

$$
-h^{\prime \prime}(r)-\frac{(2 n+1)}{r} h^{\prime}(r) \leq 2 \widetilde{\mu}_{n} h(r) \text {. }
$$

Multiplying (A.14) with the integral kernel of the resolvent of the operator on the left side of this inequality and integrating, we can proceed as in [11, Lemma 2] to arrive at (A.12).

We now are able to give the proof of Theorem 1 .

Proof of Theorem 11. We start by estimating the difference of $E_{n+1}(g)$ and $E_{n}(g)$. By analogous considerations as in [11, Eqs. (3.2) and (3.7)-(3.11)] we have, using Lemma 7 in replacement of [11, Lemma 2],

$$
\begin{aligned}
& E_{n+1}(g)-E_{n}(g) \leq(2 n+1)\left(E_{1}(g)-E_{0}(g)\right) \\
& \quad \leq(2 n+1) 2 \pi e \sup _{0<m<1}\left\|j_{m}\right\|_{\infty} \max \left\{3,1+\ln \left(\frac{\widetilde{\mu}_{m}}{2 \pi\left\|j_{m}\right\|_{\infty}}\right)\right\} .
\end{aligned}
$$


We now use the bounds (A.6) and (A.3) in order to estimate $\left\|j_{m}\right\|_{\infty}$. Our assumptions on the external potential $V$ imply that, if $\delta_{n}(R)=O(1)$,

$$
\mu_{n} \sim g^{1 /(1+2 / s+1 / p)} \quad \text { and } \quad R \sim \mu_{n}^{1 / p}
$$

for large $g$, uniformly in $n$ for bounded $n$. Therefore

$$
E_{n+1}(g)-E_{n}(g) \leq O\left(g^{-\frac{2}{s}(2+2 / s+1 / p)} \ln (g)\right) \stackrel{g \rightarrow \infty}{\longrightarrow} 0 .
$$

Note that the minimum of the GP functional over all functions with angular momentum $n$ is given by $E_{n}(g)-n|\Omega|$, and hence, for any fixed given $N$, all functions with angular momentum $<N$ cannot be GP minimizers if

$$
\min _{0 \leq n<N}\left\{E_{n}(g)-n|\Omega|\right\}>\min _{n \geq N}\left\{E_{n}(g)-n|\Omega|\right\} .
$$

This is fulfilled if

$$
|\boldsymbol{\Omega}|>\max _{0 \leq n<N} \frac{E_{N}(g)-E_{n}(g)}{N-n},
$$

which holds true, for any given fixed $N$ and $\boldsymbol{\Omega} \neq \mathbf{0}$, for large enough $g$ by (A.17).

It remains to show that for given $\boldsymbol{\Omega}$ there exists an $N_{\boldsymbol{\Omega}}$, independent of $g$, such that all functions with angular momentum $\geq N_{\Omega}$ cannot be GP minimizers. We do this by showing that they are unstable, in the sense that a small perturbation lowers the energy. This can be done by a similar calculation as in the two-dimensional case [11, using the estimates of Lemmas 517 above. We will only sketch the argument here, mainly pointing out the difference to the two-dimensional case.

In order to show instability, one has to show that the second derivative of the GP functional at some given stationary state $\phi(\boldsymbol{x})=f_{n}(r, z) e^{i n \varphi}$ is negative in some direction. More precisely,

$$
Q(w) \equiv\left\langle\left. w\left|H_{0}+16 \pi g\right| f_{n}\right|^{2}-\widetilde{\mu}_{n}+n|\Omega| \mid w\right\rangle+8 \pi g \Re \int \phi^{* 2} w^{2}<0
$$

for some $w$ with $\langle\phi \mid w\rangle=0$. Here $\Re$ denotes the real part of a complex number. We will choose an $w$ that, when added to the function $f_{n}(r, z) e^{i n \varphi}$, effectively splits the central vortex of degree $n$ into $n$ different vortices of degree 1 that are arranged symmetrically around the axis $r=0$. The idea is that for large enough $n$ a splitting of a central vortex like this should lower 
the energy of the GP functional, and therefore the second derivative given by $Q(w)$ should be negative.

Let $n \geq 1$, and let $w_{1} \in H^{1}\left(\mathbb{R}^{2}\right)$ be a radial function, with $\left\|w_{1}\right\|_{2}=1$ and support in a ball of radius 1 . Let $v \in H^{1}(\mathbb{R})$, with $\|v\|_{2}=1$, and choose as a trial function for $Q$

$$
w(r, z)=\left(2 c_{n}^{2} \widetilde{\mu}_{n}\right)^{1 / 2} w_{1}\left(\left(2 c_{n}^{2} \widetilde{\mu}_{n}\right)^{1 / 2} r\right) \frac{1}{L^{1 / 2}} v(z / L),
$$

with $c_{n}$ given in (A.13), and $L \geq 1$. Note that $c_{n}=O\left(n^{-1 / 2}\right)$ for large $n$ and, by (1.2) and (A.1),

$$
\widetilde{\mu}_{n} \geq \inf _{\boldsymbol{x}}\left\{\frac{n^{2}}{r^{2}}+V(\boldsymbol{x})\right\} \geq n \omega-C_{\omega}
$$

for any $\omega<\Omega_{c}$ and some constant $C_{\omega}$ depending only on $\omega$. Therefore $c_{n}^{2} \widetilde{\mu}_{n} \geq$ const. independent of $n$ and $g$. Using (2.8) this implies that

$$
\langle w|V| w\rangle \leq \text { const. } L^{p} .
$$

Denoting $T_{r}=\left\langle w_{1}\left|-\Delta_{r}\right| w_{1}\right\rangle, T_{z}=\left\langle v\left|-\partial^{2} / \partial z^{2}\right| v\right\rangle$ and $M_{n}=\int d^{2} r r^{2 n} w_{1}$, we get the bound (note that the last term in (A.20) is zero for the $w$ under consideration, since $n \geq 1$ )

$$
Q(w) \leq n|\Omega|-\widetilde{\mu}_{n}+2 c_{n}^{2} \widetilde{\mu}_{n} T_{r}+T_{z}+\text { const. } L^{p}+16 \pi \frac{g}{L}\left\|r^{-2 n} j_{n}(r)\right\|_{\infty} M_{n} .
$$

For some fixed $\varepsilon>0$ and $n$ large enough, choose $L$ such that the right side of (A.23) equals $\varepsilon \widetilde{\mu}_{n}$. For this choice of $L$,

$$
\left\|r^{-2 n} j_{n}\right\|_{\infty} \leq \widetilde{C}_{\varepsilon} \frac{L}{g} \widetilde{\mu}_{n}
$$

for some constant $\widetilde{C}_{\varepsilon}$ depending only on $\varepsilon$, as can be seen from Lemmas 15 and the fact that, for large $\widetilde{\mu}_{n}, R \sim \widetilde{\mu}_{n}^{1 / p}$ if $\delta_{n}(R)=O(1)$ in (A.5), again by our assumptions (2.8) and (2.9) on $V$. Using these estimates in (A.24) we obtain

$$
Q(w) \leq n|\Omega|-\widetilde{\mu}_{n}\left(1-\varepsilon-2 c_{n}^{2} T_{r}-16 \pi \widetilde{C}_{\varepsilon} M_{n}\right)+T_{z} .
$$

Since $|\Omega|<\Omega_{c}$ by assumption, and both $c_{n}^{2}$ and $M_{n}$ tend to zero as $n \rightarrow \infty$, we can choose $\varepsilon$ appropriately to conclude from (A.22) that the right side of (A.26) is negative for $n$ large enough. 
We have thus shown that for some $N_{\Omega}$, independent of $g$, all minimizers of the GP functional restricted to eigenfunctions of the angular momentum $\boldsymbol{\Omega} \cdot \boldsymbol{L}$ with eigenvalue $n \geq N_{\boldsymbol{\Omega}}$ are unstable and therefore cannot be absolute minimizers of the GP functional. Together with the result A.19) above this proves Theorem [1]

Acknowledgments. Helpful discussions with Elliott Lieb and Jakob Yngvason, as well as financial support by the Austrian Science Fund in the from of an Erwin Schrödinger fellowship, are gratefully acknowledged.

\section{References}

[1] E.A. Cornell and C.E. Wieman, Bose-Einstein condensation in a dilute gas, the first 70 years and some recent experiments, in: Les Prix Nobel 2001 (The Nobel Foundation, Stockholm, 2002), pp. 87-108. Reprinted in: Rev. Mod. Phys. 74, 875-893 (2002); Chem. Phys. Chem. 3, 476-493 (2002)

[2] W. Ketterle, When atoms behave as waves: Bose-Einstein condensation and the atom laser, in: Les Prix Nobel 2001 (The Nobel Foundation, Stockholm, 2002), pp. 118-154. Reprinted in: Rev. Mod. Phys. 74, 1131-1151 (2002); Chem. Phys. Chem. 3, 736-753 (2002)

[3] K.W. Madison, F. Chevy, W. Wohlleben, and J. Dalibard, Vortex Formation in a Stirred Bose-Einstein Condensate, Phys. Rev. Lett. 84, 806-809 (2000)

[4] J.R. Abo-Shaeer, C. Raman, J.M. Vogels, and W. Ketterle, Observation of Vortex Lattices in Bose-Einstein Condensates, Science 292, 476-479 (2001)

[5] A.L. Fetter and A.A. Svidzinsky, Vortices in a trapped dilute BoseEinstein condensate, J. Phys.: Condens. Matter 13, R135-R194 (2001)

[6] F. Dalfovo, S. Giorgini, L.P. Pitaevskii, and S. Stringari, Theory of BoseEinstein condensation in trapped gases, Rev. Mod. Phys. 71, 463-512 (1999) 
[7] E.H. Lieb, R. Seiringer, and J. Yngvason, Bosons in a Trap: A Rigorous Derivation of the Gross-Pitaevskii Energy Functional, Phys. Rev. A 61, 043602-1-13 (2000)

[8] E.H. Lieb and R. Seiringer, Proof of Bose-Einstein Condensation for Dilute Trapped Gases, Phys. Rev. Lett. 88, 170409-1-4 (2002)

[9] E.H. Lieb, R. Seiringer, and J. Yngvason, Superfluidity in Dilute Trapped Bose Gases, Phys. Rev. B 66, 134529-1-6 (2002)

[10] E.H. Lieb and J. Yngvason, The Ground State Energy of a Dilute TwoDimensional Bose Gas, J. Stat. Phys. 103, 509-526 (2001)

[11] R. Seiringer, Gross-Pitaevskii Theory of the Rotating Bose Gas, Commun. Math. Phys. 229, 491-509 (2002)

[12] E.H. Lieb, R. Seiringer, and J. Yngvason, A Rigorous Derivation of the Gross-Pitaevskii Energy Functional for a Two-Dimensional Bose Gas, Commun. Math. Phys. 224, 17-31 (2001)

[13] E.H. Lieb, R. Seiringer, and J. Yngvason, Poincaré Inequalities in Punctured Domains, Preprint arXiv:math.FA/0205088, Ann. Math. (in press)

[14] M. Reed and B. Simon, Methods of Modern Mathematical Physics IV. Analysis of Operators, Academic Press (1978)

[15] J. Avron, I. Herbst, and B. Simon, Schrödinger Operators with Magnetic Fields. I. General Interactions, Duke Math. J. 45, 847-883 (1978)

[16] E.H. Lieb and J. Yngvason, Ground State Energy of the Low Density Bose Gas, Phys. Rev. Lett. 80, 2504-2507 (1998)

[17] E.H. Lieb, R. Seiringer, J.P. Solovej, and J. Yngvason, The Ground State of the Bose Gas, in: Current Developments in Mathematics, 2001, 131-178, International Press, Cambridge (2002)

[18] E.H. Lieb and B. Simon, The Thomas-Fermi Theory of Atoms, Molecules and Solids, Adv. Math. 23, 22-116 (1977)

[19] F.J. Dyson, Ground-State Energy of a Hard-Sphere Gas, Phys. Rev. 106, 20-26 (1957) 
[20] E.H. Lieb, The Stability of Matter: From Atoms to Stars, Bull. Amer. Math. Soc. 22, 1-49 (1990) 